\title{
An enhanced sum rate in the cluster based cognitive radio relay network using the sequential approach for the future Internet of Things
}

\author{
Md Sipon Miah ${ }^{1,2^{*}}$ (D), Michael Schukat ${ }^{1 \dagger}$ and Enda Barrett ${ }^{1+}$
}

\section{${ }^{*}$ Correspondence:}

m.miah1@nuigalway.ie

${ }^{\dagger}$ Miah Md Sipon, Michael

Schukat and Enda Barrett

contributed equally to this work

${ }^{1}$ Department of Information

Technology, National

University of Ireland Galway,

University Road, Galway H91

TK33, Ireland

Full list of author information is available at the end of the article

\begin{abstract}
The cognitive radio relay plays a vital role in cognitive radio networking (CRN), as it can improve the cognitive sum rate, extend the coverage, and improve the spectral efficiency. However, cognitive relay aided CRNs cannot obtain a maximal sum rate, when the existing sensing approach is applied to a CRN. In this paper, we present an enhanced sum rate in the cluster based cognitive radio relay network utilizing a reporting framework in the sequential approach. In this approach a secondary user (SU) extends its sensing time until right before the beginning of its reporting time slot by utilizing the reporting framework. Secondly all the individual measurement results from each relay aided SU are passed on to the corresponding cluster head $(\mathrm{CH})$ through a noisy reporting channel, while the $\mathrm{CH}$ with a soft-fusion report is forwarded to the fusion center that provides the final decision using the n-out-of-k-rule. With such extended sensing intervals and amplified reporting, a better sensing performance can be obtained than with a conventional non-sequential approach, therefore making it applicable for the future Internet of Things. In addition, the sum rate of the primary network and CCRRN are also investigated for the utilization reporting framework in the sequential approach with a relay using the $n$-out-of-k rule. By simulation, we show that the proposed sequential approach with a relay (Lemma 2) provides a significant sum rate gain compared to the conventional non-sequential approach with no relay (Lemma 1) under any condition.
\end{abstract}

Keywords: Cognitive radio, Channel sensing, Amplify and forward relay, Fusion center, Non-sequential approach, Sequential approach, Internet of Things

\section{Introduction}

\section{Motivation}

Cognitive radio (CR) is a promising wireless communication technology that improves spectrum band utilization by using it more flexibly, intelligently and efficiently [1]. When a secondary user (SU) supports the spectrum sensing capability in a cognitive radio network (CRN), they can discover and select frequency bands that are most suitable for use. A precondition of SU access is that SUs opportunistically use the spectrum allocated to the primary network (PN) without causing harmful interference to the primary user 
(PU) and instantly vacate the allocated spectrum when the PU appears. For this reason, devising optimal spectrum sensing strategies is one of the most important research topics in a CRN [2]. Thus the performance of a CRN significantly depends on the capability of the SU transmitters to sense the primary channel.

Internet of Things (IoT) is a promising technology that allows communications among sensor nodes, a continuous exchange of context between sender and receiver, and the ability to join and leave the network spontaneously [3-6]. IoT has two essential properties: self-adaptation and self-organization. However, the great challenges for future IoT based multimedia applications are the spectrum scarcity problem, high implementation cost, high energy consumption, and low sum rate as compared with more general radio platforms due to the rapid increase in the number of wireless devices present in future IoT [6] systems. In order to support the applicability of CR for future IoT, the cluster based cognitive radio relay network (CCRRN) which utilises reporting frameworks is a promising approach. We propose a sequential approach with a relay which can be enhanced to more optimally consume the available spectrum and improve the sum rate in transmission.

Spectrum sensing plays a vital role in CRNs and can be broadly classified into two main categories: (1) non-cooperative spectrum sensing (NCSS) $[7,8]$ and (2) cooperative spectrum sensing (CSS) $[9,10]$. In (1), some of the most important techniques in the literature are, matched filter detection [11], cyclostationary based detection [12] and energy detection (ED) $[13,14]$. The matched filter is a faster and more optimal sensing technique which depends on the prior knowledge of the PU transmission including modulation type, bandwidth, carrier frequency, pulse shaping and frame format, etc. In cyclostationary based detection, overall performance is good, but it still requires partial knowledge of the PU's signal such as the properties of cyclic frequencies. On the other hand, the ED is considered as an attractive method due to the fact that it does not require any prior knowledge of the incumbent signal, its easy implementation and low complexity. However, the spectrum sensing accuracy is compromised because of fading, shadowing, uncertainty and the hidden terminal problem [15-17]. In (2), it reduces sensing delay and improves sensing accuracy as it mitigates against the effects of fading, shadowing, uncertainty and the hidden terminal problem. It can be broadly classified into three main categories: (a) centralized, which declares one SU as a central node to make a final decision, (b) distributed, which makes a final decision through the linear combination of independent decisions and (c) relay-based spectrum sensing, which obtains higher PU detection probability due to the presence of a relay to assist the SU with lower PU detection probability [18]. In the centralized case, each SU performs local sensing independently and then forwards the sensing results to the fusion center (FC) through the noise free reporting channel between the SUs and the FC. The FC makes a final decision according to some fusion rules. These fusion rules can be classified as soft decision fusion (SDF) or hard decision fusion (HDF).

When we consider noisy reporting channels, the advantages of cooperative sensing can be limited [19-22]. To mitigate against this problem [23-25], a cluster based cooperative sensing scheme divides all the SUs into a number of clusters and selects the most favorable $\mathrm{SU}$ in each cluster as a cluster head $(\mathrm{CH})$ to report sensing results. This can dramatically lessen the performance deterioration caused by the fading of the wireless 
channels. In these schemes, the $\mathrm{SU}$ selected as the $\mathrm{CH}$ has to fuse sensing data from all the cluster members (the SUs in this cluster). However, the existing spectrum sensing strategies have been evaluated by using rigid sensing time slots in a CRN. This means that each SU's reporting time slot and the $\mathrm{CH}$ reporting time slot offer no contribution to spectrum sensing, while $\mathrm{SU}$ sensing and reporting times and $\mathrm{CH}$ reporting times are in different time slots.

\section{Contributions}

In this paper, we have achieved the following major contributions:

- We propose an enhanced sum rate in the CCRRN using the sequential approach with a relay for future IoT systems, and formulate the sensing performance and the sum rate maximization problem in the conventional non-sequential approach with no relay [38]. The proposed sequential approach with and without a relay, requires us to use a utilization reporting framework to solve the maximization problem for the conventional non-sequential approach.

- The proposed sequential approach in the CCRRN with and without a relay, is considered a noisy reporting channel between the $\mathrm{SU}$ and the $\mathrm{CH}$ due to $\mathrm{SU}$ members in the cluster often being at a distance from each other.

- We propose an efficient reporting mechanism in which each SU achieves a longer/ flexible sensing time slot to sense the PU signal due to a utilization reporting framework that employs the sequential approach in the CCRRN with a relay.

- We empirically examine the sensing performances at $\mathrm{SUs}, \mathrm{CHs}$ and the $\mathrm{FC}$ by extending the sensing time slots with and without a relay using the SDF scheme i.e., ' $n-$ out - of $-k-$ rule'.

- Based on the false alarm and detection probabilities, the sum rate of the PN and CCRRN are analyzed using the conventional non-sequential approach in the CCRRN with no relay and the proposed sequential approach in the CCRRN with and without a relay.

- We calculate the optimal false alarm probability (Lemma 3) which enhances the sum rate in the proposed sequential approach in the CCRRN with and without a relay; compared with the conventional non-sequential approach in the CCRRN with no relay.

\section{Organization}

The remainder of the paper is organized as follows. The general motivation and the background of this paper is summarized in the "Related works" section. "System model" section describes the system model which consists of a PN and a CCRRN. "Energy detection technique" section describes how each SU estimates its own measured energy from the PU signal. "The conventional non-sequential approach in the CCRRN" section describes the conventional non-sequential approach in the CCRRN where each SU underutilizes the reporting framework. "The proposed sequential approach in the CCRRN utilizing the reporting framework" section describes the sensing performance and the sum rate analysis where each SU utilizes the reporting framework. The simulation parameters of the proposed approach and comparisons are given in the "Simulation 
Table 1 Main parameters

\begin{tabular}{|c|c|}
\hline Parameters & Meaning \\
\hline$H\left(H_{0} / H_{1}\right)$ & Hypotheses (absent/present) \\
\hline$\tau_{\varsigma}\left(\tau_{s}^{c o n} / \tau_{s}^{p r o p}\right)$ & $\begin{array}{l}\text { Sensing time slot (in the conventional non-sequential approach/ in the proposed sequential } \\
\text { approach) }\end{array}$ \\
\hline$\tau_{r}\left(\tau_{r, S U} / \tau_{r, C H}\right)$ & Reporting time slot (reporting time slot at the SU/CH) \\
\hline$P_{f, k}^{c o n}(0)$ & $\begin{array}{l}\text { Probability of false alarm in the conventional non-sequential approach with no relay at the } k \text { th } \\
\text { cluster }\end{array}$ \\
\hline$P_{f, k}^{p r o p(1)}$ & Probability of false alarm in the proposed sequential approach with a relay at the $k$ th cluster \\
\hline$P_{f, k}^{p r o p(2)}$ & Probability of false alarm in the proposed sequential approach with no relay at the kth cluster \\
\hline$P_{d, k}^{c o n}(0)$ & $\begin{array}{l}\text { Probability of detection in the conventional non-sequential approach with no relay at the } k \text { th } \\
\text { cluster }\end{array}$ \\
\hline$P_{d, k}^{p r o p(1)}$ & Probability of detection in the proposed sequential approach with a relay at the $k$ th cluster \\
\hline$P_{d, k}^{p r o p(2)}$ & Probability of detection in the proposed sequential approach with no relay at the $k$ th cluster \\
\hline$P_{f, k}^{*}$ & The optimal probability of false alarm at the $k$ th cluster \\
\hline$\lambda_{k}^{c o n}$ & Decision threshold in the conventional non-sequential approach with no relay at the $k$ th cluster \\
\hline$\lambda_{k}^{\text {prop }}$ & Decision threshold in the proposed sequential approach with a relay at the $k$ th cluster \\
\hline$\hat{P_{f, F C}^{m}} / P_{d, F C}^{m}$ & Global decision under binary hypotheses at the $\mathrm{FC}$, here $m$ stands for $(0,1,2)$ \\
\hline$P_{t} / \gamma_{j} / \gamma_{k}$ & $\begin{array}{l}\text { Amplify power at each SU/ the signal to noise ratio at the } j \text { th } \mathrm{SU} / \text { the signal to noise ratio at the } \\
k \text { th cluster }\end{array}$ \\
\hline$Q(\cdot) / Q^{-1}(\cdot)$ & Gaussian/ inverse Gaussian tail function \\
\hline$\delta_{d}$ & $\begin{array}{l}\text { The network end-to-end delay in the conventional non-sequential approach with no relay/the } \\
\text { proposed sequential approach with and without a relay }\end{array}$ \\
\hline
\end{tabular}

results and discussion" section. We compare our proposed approach with other existing approaches which demonstrates better gain in terms of detection performance and sum rate maximization with low complexity. Finally, our conclusions and future work are addressed in the "Conclusions and future works" section. In order to make the paper more readable, the main parameters are listed in Table 1.

\section{Related works}

CCRRNs with a relay scheme play an important role in utilizing the reporting framework. Thus, several publications are listed below that provide the background and recent research contributions of a sequential approach with and without a relay, and nonsequential approach with no relay schemes. The analysis of NCSS in a CRN is presented in $[7,8]$. An analysis of CSS in a CRN is presented in [9, 10]. An efficient transmission mode selection based on reinforcement learning for cooperative CRNs is studied in [10]. It is shown that the spectrum sensing accuracy is compromised because of fading, shadowing, uncertainty and the hidden terminal problem [15-17]. In order to mitigate the fading problem, the relay based spectrum sensing in the CRN is studied in [18]. The advantages of CSS can be limited due to noisy reporting channels, which are studied in [19-22]. To mitigate against this problem, cluster based CSS schemes are studied in [23-25]. Effective implementation of security based algorithmic approaches in mobile ad-hoc network and stochastic approaches for dynamic power management in wireless sensor network (WSN) are studied in [26, 27]. In a CRN with a large number of SUs, CSS requires many reports from SUs to the FC through the control channel, which can 
result in overhead traffic of the control channel. Some methods have been proposed to solve these problems with CSS, such as cluster-based CSS [28] and sequential CSS [29-31]. Vu-Van et al. [32] proposed a cluster based sequential CSS scheme for CRNs in order to significantly reduce the number of direct reports from SUs to the FC while keeping the similar sensing performance to that of the conventional CSS scheme. In this scheme, each SU performs local sensing independently and then forwards the sensing of hard decisions to the corresponding $\mathrm{CH}$ through the noise free reporting channel. All CHs forward their hard decisions to the corresponding FC through the noise free reporting channel. However, the sensing performance of this scheme may be decreased due to fading effects of reporting channels in real environments. Therefore, an efficient reporting mechanism is needed in the cluster based CSS scheme for enhanced spectrum sensing and sum rate maximization. Moreover, the sensing performance is also decreasing when we are using the HDF scheme, i.e., 'OR - rule' and 'AND - rule'. Therefore, the SDF scheme is needed in the cluster based CSS for further enhanced spectrum sensing and sum rate maximization. In [19-22,33], with the objective of maximizing the sum rate of the CRN, a cluster-based CSS is proposed to obtain a proper assignment policy. However, the reporting channels are considered as error-free. Moreover, the sum rate was not analyzed as a utilization reporting framework. In [23-25, 34], the authors propose a cluster-based CSS strategy to maximize an achievable sum rate scheme in the non-error-free environment. However, the sum rate was not analyzed as a utilization reporting framework. Hung et al. [35] proposed cognitive cooperative networks with a cluster based relaying scheme in which the secondary base station (SBS) transmits signals to multiple secondary receivers (SU-Rx) through the help of multiple secondary relays. However, the reporting channel between the SBS and SU-Rx is noise free. Yu $H[36,37]$ proposed the optimal channel sensing maximizing sum rate in CRNs with multiple SUs with the capacity of the CRN being analyzed. Moreover, some interesting characteristics including asymptotic results were observed. However, the sum rate was not analyzed as a utilization reporting framework approach in the CCRRN. Miah et al. [38] proposed maximization of the sum rate in amplify and forward (AF)-cognitive radio networks using the superposition approach with the sum rate of CRNs being analyzed. However, the sum rate was not analyzed based on the optimal probability of false alarm. Moreover, the sequential approach was not analyzed which would be a more favorable approach due to the limited reporting control channel.

\section{System model}

The proposed system model consists of a PN and a CCRRN as shown in Fig. 1 in which the SU is denoted as an unlicensed user that opportunistically accesses the spectrum of the PU without causing interference, whilst the PU remains the licensed user of the spectrum. The PN consists of PUs, i.e., primary transmitters and receivers. The operation of the PU is considered to be time division multiplexing access (TDMA). We assume that for each slot the PU transmitter sends data to its receiver independently with a probability $\rho \in[0,1]$, which is defined as the primary activity factor. On the other hand, it is assumed that the $\mathrm{SU}$ network is also a time slot-based network. The CCRRN consists of $N$ SUs, i.e., secondary user transmitters and receivers that will simply act in an AF relay manner, $K \mathrm{CHs}$ and a FC where the $N$ SUs are grouped into a cluster controlled by a $\mathrm{CH}$ 


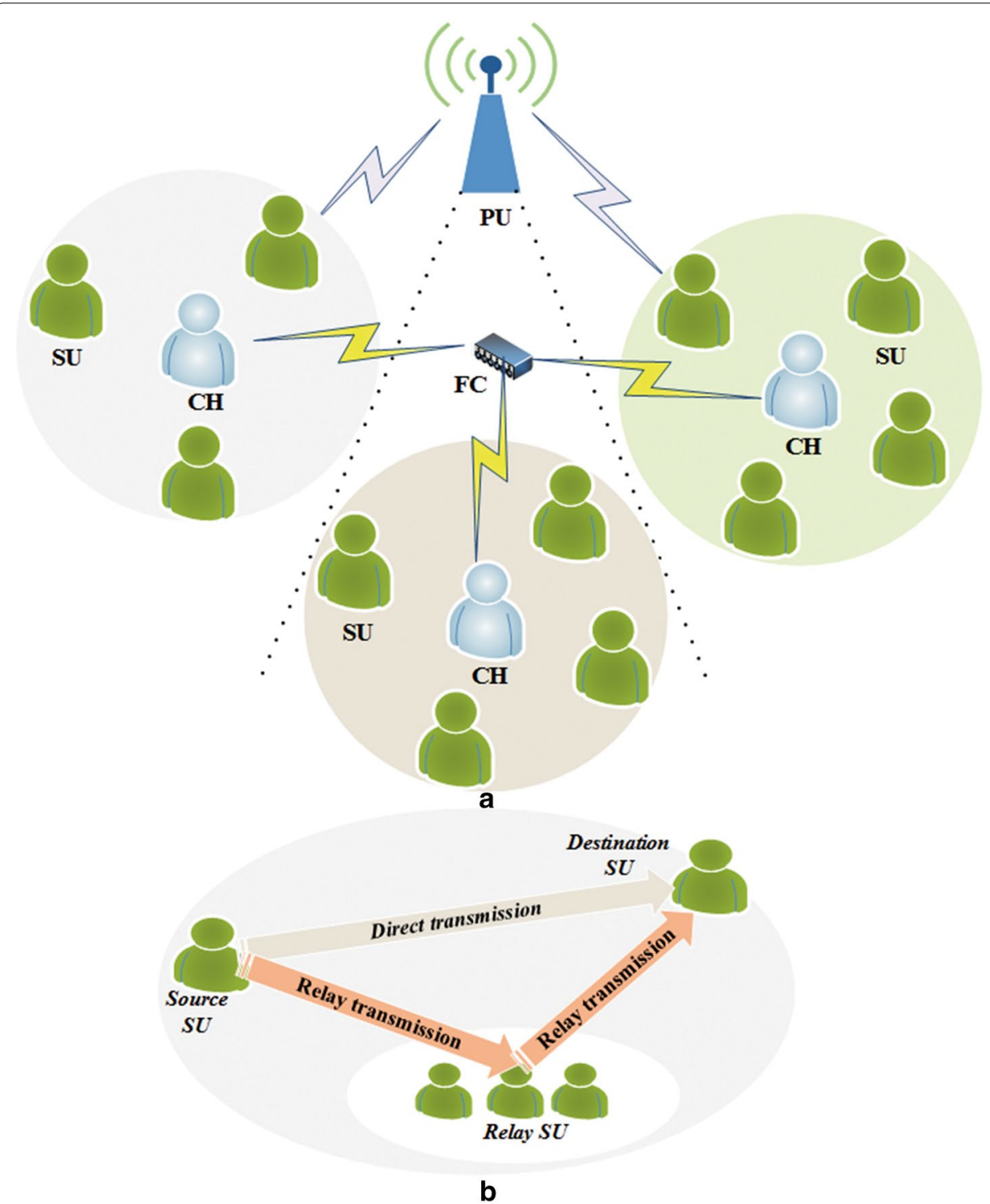

Fig. 1 The proposed system model: a all SUs build up a cluster based on the LEACH-C protocol; b SU interaction with and without a relay

based on a low energy adaptive clustering hierarchy-centralized (LEACH-C) protocol [39-42] as shown in Fig. 1a. The process of the LEACH-C protocol is the build up of rounds; each round consists of two phases as follows: (1) the setup phase and (2) the steady state phase.

In (1), each SU sends a 'request - message' $=\left(S U_{I D}, L O C(C), S N R\right)$ to the FC. Based on this 'request - message', the FC determines $\mathrm{CHs}$ among all SUs, while the remaining SUs will act as cluster members. After the $\mathrm{CHs}$ are determined, the $\mathrm{FC}$ broadcasts a 'reply-message' $=\left(C_{I D}, S Y N(T)\right)$. If the SUs CH ID matches its own ID, then the $\mathrm{SU}$ is a $\mathrm{CH}$; otherwise, the $\mathrm{SU}$ is a cluster member and goes to sleep. Here $S U_{I D}, C H_{I D}$, $L O C(C), S N R$ and $S Y N(T)$ stands for the identification number at the SU, the identification number at the $\mathrm{CH}$, the current $\mathrm{SU}$ location, the signal to noise ratio and the 
time synchronization, respectively. Moreover, the primary and secondary users are synchronized.

In (2), the SUs start to share their measurement of the received PU signal to the corresponding $\mathrm{CH}$, and then the $\mathrm{CH}$ collects their measurements and makes the cluster decision and relays it to the FC. Afterwards, the FC combines the received cluster decisions to make the global decision and then broadcasts it back to all $\mathrm{CHs}$ and the $\mathrm{CHs}$ send it to their cluster members.

In the proposed CCRRN with no relay scheme, the source SU sends the data to the destination SU during the time slot using a direct link. For the proposed CCRRN with a relay scheme, the source $\mathrm{SU}$ sends the data to the destination $\mathrm{SU}$ during the time slot and the relay SU receives the data on the same time slot due to the broadcast nature of communication, and then finally forwards the data to the destination SU in the manner of the AF protocol as shown in Fig. 1b.

Let $H_{1}$ and $H_{0}$ be the hypotheses representing the presence and absence of primary signals, respectively. Under this binary hypothesis testing problem, the spectrum sensing can be formulated as follows

$$
\left\{\begin{array}{l}
H_{1}: \text { if PU is present } \\
H_{0}: \text { if PU is absent }
\end{array}\right.
$$

Depending on the packet transmission of the PU, the received signals of the $j$ th $\mathrm{SU}$ can be formulated as follows

$$
\left.y_{j}(l)\right|_{l=1} ^{L}=\theta h_{j}(l) x(l)+w_{j}(l)
$$

where $\theta=1$ denotes the presence of the PU while $\theta=0$ denotes the absence of the PU, $y_{j}(l)$ denotes the sensing signal received by the $j$ th $\mathrm{SU}, h_{j}(l)$ is the channel gain between the $j$ th SU and the PU with $h=\sum_{j=1}^{N} h_{j}$. Moreover, $x(l)$ is the PU transmit signal which is modulated on a binary phase shift keying (BPSK) signal with power $p_{x}^{2}, w_{j}(l)$ is a circularly symmetric complex Gaussian (CSCG) noise with the variance of $\sigma_{w, j}^{2}$ by the $j$ th SU, and $L$ is the number of samples.

Each SU will simply act in an AF manner, the $N$ SUs relay their individual measurements of the PUs signal, $y_{j}(l)$ to the $k$ th corresponding $\mathrm{CH}$ through a noisy control channel in the sequential manner. At the $\mathrm{CH}$, the relay signal received by the corresponding $\mathrm{CH}$ from the $j$ th $\mathrm{SU}$ will be given as follows

$$
r_{j}(l)= \begin{cases}\sqrt{P}_{t} g_{j}(l) y_{j}(l)+z_{j}(l) ; & \text { with AF manner } \\ g_{j}(l) y_{j}(l)+z_{j}(l) ; & \text { without AF manner }\end{cases}
$$

where $\sqrt{P}_{t}$ denotes the transmit power of each SU relay, $g_{j}$ denotes the amplitude of the channel gain of the $j$ th SU and $k$ th $\mathrm{CH}$ link, i.e., $g=\sum_{j=1}^{N} g_{j}(l)$, and $z_{j}(l)$ is the noisy reporting channel of the $j$ th $\mathrm{SU}$ and $k$ th $\mathrm{CH}$ link which has zero mean and an additive white Gaussian noise with variance, i.e., $\sigma_{z, j}^{2}$. Then the signal to noise ratio (SNR) at the $k$ th $\mathrm{CH}$ is given as follows

$$
\gamma_{k}=\sum_{j=1}^{N} \gamma_{j}=\frac{p_{x}^{2}}{\sigma_{w+r}^{2}=\sum_{j=1}^{N}\left(\sigma_{w, j}^{2}+\sigma_{z, j}^{2}\right)}
$$




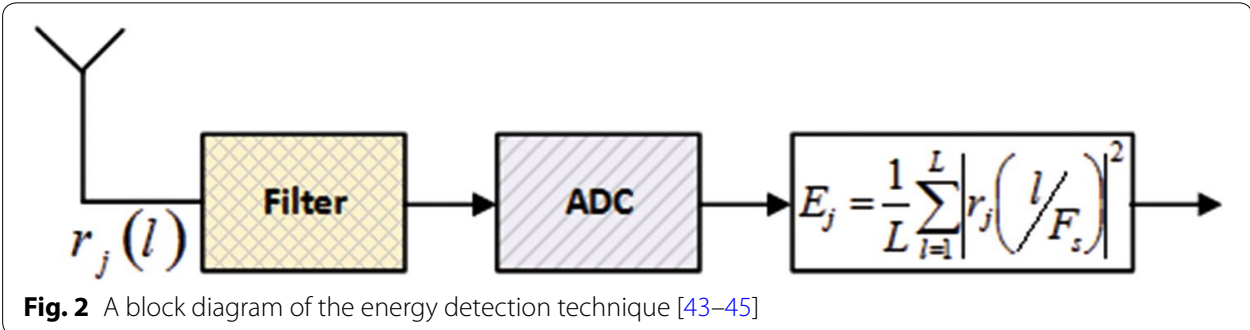

where $\gamma_{k}$ is the SNR at the $k$ th $\mathrm{CH}$, which is defined as the ratio of the signal power to noise power.

\section{Energy detection technique}

The ED technique [43-45] is widely used in the spectrum sensing of the SU, because it can be implemented easily without acquiring any prior information of the PU signal. We assume that each SU transmitter senses the PU signal using the ED technique. The structure of the channel sensing process at the SU transmitter employing the ED is shown in Fig. 2.

At the particular cluster with $N$ SUs, the sensing result, $r_{j}(l)$ received by the $j$ th $\mathrm{SU}$ transmitter is the signal power in a particular frequency in the time domain, a band-pass filter is applied to the received signal, then the output of this filter is transformed by an analog-to-digital converter (ADC), which will be individually averaged and squared using the ED to estimate its own measured energy as given by

$$
E_{j}=\frac{1}{L} \sum_{l=1}^{L}\left|r_{j}\left(\frac{l}{F_{s}}\right)\right|^{2}
$$

where $r_{j}\left(\frac{l}{F_{s}}\right)$ is the lth sample of a received signal by the $j$ th $\mathrm{SU}$ at the $\mathrm{CH}$ which is defined as $L=\tau_{s} F_{s}$, here $\tau_{s}$ is the sensing duration which is commonly used by all SUs to sense the PU signal and $F_{s}$ is the sampling frequency of the PU signal.

The probability distribution function (PDF) can be approximated as a Gaussian random variable using the central limit theorem (CLT) as follows

$$
\begin{cases}\aleph\left(\mu_{0}=\sum_{j=1}^{N} \mu_{0, j},\right. & \left.\sigma_{0}^{2}=\sum_{j=1}^{N} \sigma_{0, j}^{2}\right) \\ \aleph\left(\mu_{1}=\sum_{j=1}^{N} \mu_{1, j},\right. & \left.\sigma_{1}^{2}=\sum_{j=1}^{N} \sigma_{1, j}^{2}\right)\end{cases}
$$

\section{Conventional non-sequential approach in the CCRRN}

In the conventional non-sequential approach with no relay, all SUs sense the PU signal at a time during the rigid sensing time slot and forward their sensing results to the $\mathrm{CH}$ during the reporting time slot. In this conventional approach, we do not utilize the reporting framework as all SUs forward their sensing results to the $\mathrm{CHs}$ in a non-sequential manner. As an example, the 2nd SU in the 1st cluster cannot utilize the rigid reporting time slot of the 1st SU for sensing the PU signal, and the 3rd SU in the 1st cluster cannot utilize the rigid reporting time slots of the previous 1st SU and 2nd SU for sensing 


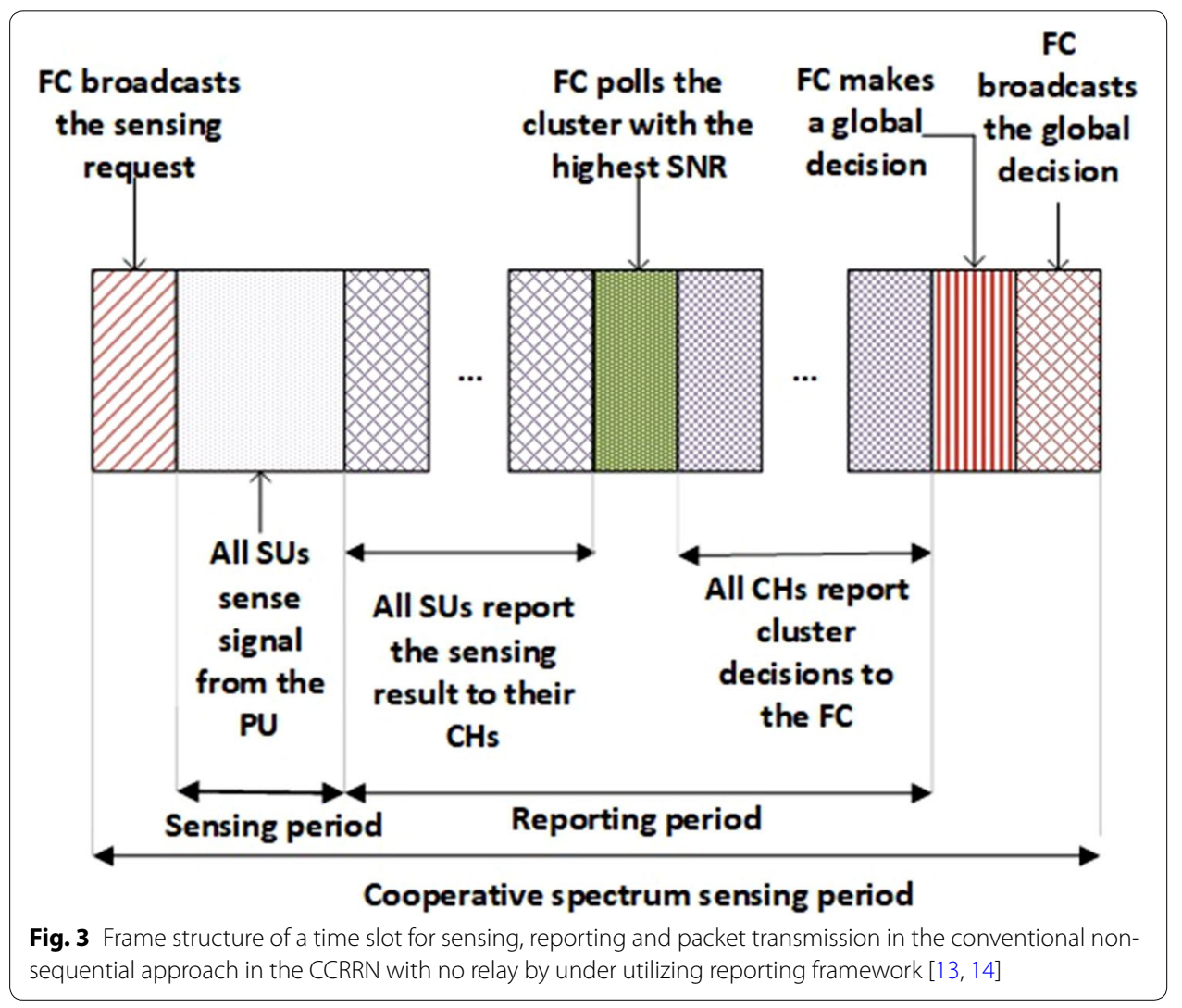

the PU signal, and so on; similarly, the 1st SU in the 2nd cluster cannot utilize the rigid reporting time slot of the 1st cluster head for sensing the PU signal, and the 1st SU in the 3rd cluster cannot utilize the rigid reporting time slots of the previous 1st cluster head and 2nd cluster head for sensing the PU signal, and so on [13]. Figure 3 shows the frame structure for the conventional non-sequential approach in the CCRRN with no relay and under utilizing the reporting framework. Under the given frame structure, all SUs sense the PU channel with a rigid sensing time slot $\tau_{s}$, as shown in Algorithm 1. During a rigid sensing time slot $\tau_{s}$, the conventional non-sequential approach in the CCRRN with no relay will not be applicable for the future IoT as the detection gain is not sufficient.

Lemma 1 When the PU signal, $x(l)$ is a BPSK-modulated signal, the channel noise between the PU and the jth $S U, w_{j}(l)$, is a CSCG, and the reporting channel between the jth SU and the kth CH is noise-free. An estimation of the received signal power is given by all SUs in the conventional non-sequential approach in the CCRRN with no relay as follows [38]

$$
E= \begin{cases}\mu_{0}=L \sigma_{w}^{2}, & \sigma_{0}^{2}=L \sigma_{w}^{4} \\ \mu_{1}=L\left(1+|h|^{2} \gamma\right) \sigma_{w}^{2}, & \sigma_{1}^{2}=L\left(1+2|h|^{2} \gamma\right) \sigma_{w}^{4}\end{cases}
$$

Proof Please see Appendix. 
Now, the $k$ th $\mathrm{CH}$ calculates a cluster decision test statistic from all the individual test statistics $\left[E_{1}, E_{2}, \ldots, E_{N}\right]$ of an individual SU and multiplies the weight-coefficient of the SU by a linear statistic combination (LSC) manner as follows

$$
\left.C_{k}\right|_{k=1} ^{K}=\sum_{j=1}^{N} \omega_{j} E_{j}
$$

where $\omega_{j}$ is the weight-coefficient assigned on the $j$ th SU at the $k$ th cluster.

Based on the Eq. (8), we can calculate the $k$ th cluster probability of false alarm $P_{f, k}^{c o n}=\operatorname{Pr}\left[H_{1} \mid H_{0}\right]$ and the probability of detection $P_{d, k}^{c o n}=\operatorname{Pr}\left[H_{1} \mid H_{1}\right]$ as given for a preselected threshold of $\lambda_{k}^{\text {con }}$

$$
\begin{aligned}
P_{f, k}^{c o n} & =\operatorname{Pr}\left[C_{k} \geq \lambda_{k}^{c o n} \mid H_{0}\right] \\
& =Q\left(\left(\frac{\lambda_{k}^{c o n}}{\sigma_{w}^{2}}-1\right) \sqrt{\tau_{s} F_{s}}\right) \\
P_{d, k}^{c o n} & =\operatorname{Pr}\left[C_{k} \geq \lambda_{k}^{c o n} \mid H_{1}\right] \\
& =Q\left(\left(\frac{\lambda_{k}^{c o n}}{\sigma_{w}^{2}}-|h|^{2} \gamma-1\right) \sqrt{\frac{\tau_{s} F_{s}}{\left(1+2|h|^{2} \gamma\right)}}\right)
\end{aligned}
$$

where $Q(t)$ denotes a Gaussian tail function which is defined as $Q(t)=\frac{1}{\sqrt{2 \pi}} \int_{t}^{\infty} e^{-\frac{x^{2}}{2}} d x$.

From Eqs. (9) and (10), we can calculate the decision threshold $\lambda_{k}^{\text {con }}$ at the $k$ th $\mathrm{CH}$ as follows

$$
\lambda_{k}^{c o n}= \begin{cases}{\left[\frac{Q^{-1}\left(P_{f, k}^{c o n}\right)}{\sqrt{\tau_{s} F_{s}}}+1\right] \sigma_{w}^{2} ;} & H_{0} \\ {\left[\frac{Q^{-1}\left(P_{d, k}^{c o n}\right)}{\sqrt{\frac{\tau_{f} F_{s}}{\left(1+2|h|^{2} \gamma\right)}}}+1+|h|^{2} \gamma\right] \sigma_{w}^{2} ;} & H_{1}\end{cases}
$$

where $Q^{-1}(t)$ denotes an inverse-Gaussian tail function.

We can calculate the sensing time in the conventional non-sequential approach in the CCRRN with no relay and guarantee the sensing performance given by the probability of false alarm and the probability of detection using Eqs. (9) and (10) as follows [38]

$$
\tau_{s}^{c o n}=\frac{1}{F_{s}|h|^{4} \gamma^{2}}\left[Q^{-1}\left(P_{f, k}^{c o n}\right)-Q^{-1}\left(P_{d, k}^{c o n}\right) \sqrt{\left(1+2|h|^{2} \gamma\right)}\right]
$$

Proposition 1 In the conventional non-sequential approach in the CCRRN with no relay, all the SUs at the kth cluster have obtained the same fixed/rigid sensing time slot which is denoted as

$$
\tau_{s}^{k j}=\tau_{s}^{c o n}
$$

where $\tau_{s}^{k j}$ denotes the flexible sensing time slot for the jth SU at the kth cluster that is equal to $\tau_{s}^{\text {con }}$ which denotes the fixed/rigid sensing time slot for all SUs. 
Proof Please see Appendix.

In Algorithm 1, all SUs are under utilizing the reporting framework as obtained fixed/ rigid sensing time slot (see line 5). Then, it computes the probability of false alarm (see line 7 ) and the probability of detection (see line 8 ) at the $k$ th cluster in the conventional non-sequential approach with no relay $\left(P_{t}=1\right)$.

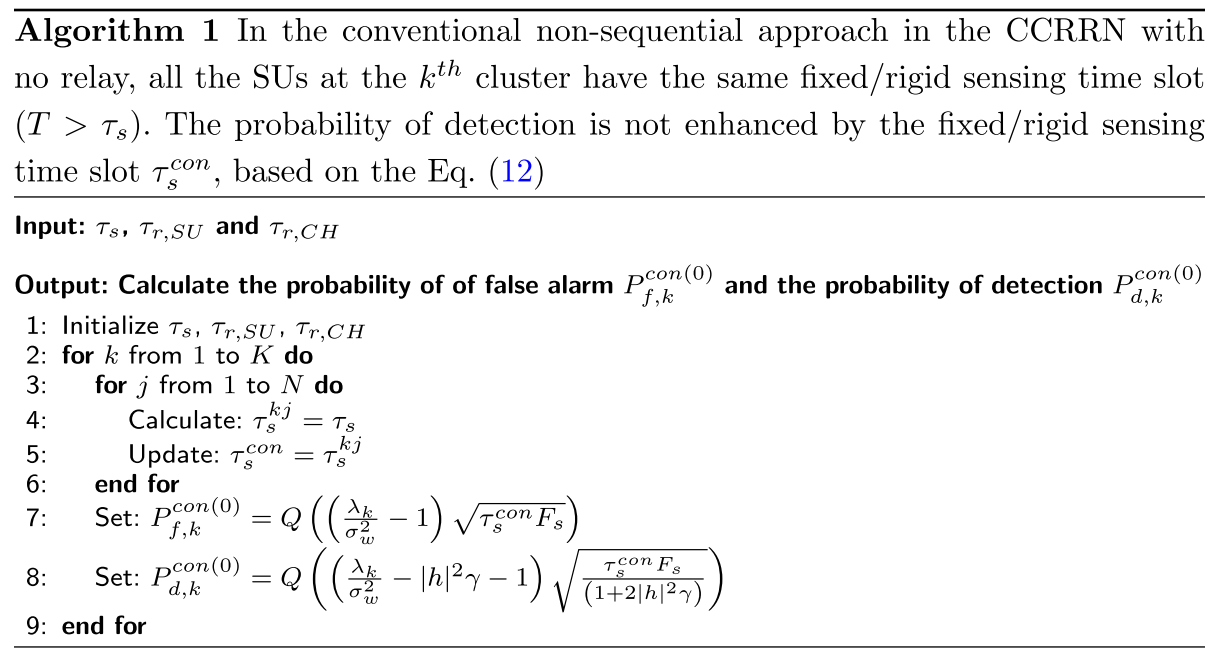

Proposed sequential approach in the CCRRN utilizing the reporting framework In the proposed sequential approach in the CCRRN, the sensing performance is discussed in the "Spectrum sensing analysis" section and the system sum rate is discussed in the "Sum rate analysis" section.

\section{Spectrum sensing analysis}

The proposed scheme utilizing the reporting framework, namely the proposed sequential approach in the CCRRN, is a promising solution for the spectrum scarcity problem [46-48] of the future IoT multimedia applications. In this approach, each SU can obtain a longer/flexible sensing time slot due to the rest of the SUs reporting time slots and the $\mathrm{CH}$ reporting time slots being combined to the longer/flexible sensing time slot for that as shown in Fig. 4 and Algorithm 2. A major challenge in the proposed sequential approach is utilizing the spectrum more efficiently which will be more applicable for the future IoT systems.

In Fig. 4, the 2nd SU in the 1st cluster can utilize the rigid reporting time slot of the 1st SU for sensing the PU signal, and the 3rd SU in the 1st cluster can utilize the fixed/rigid reporting time slots of the previous 1st SU and 2nd SU for sensing the PU signal, and so on. Moreover, the 1st $\mathrm{SU}$ in the 2nd cluster can utilize the rigid reporting time slot of the 1st cluster head $\left(\mathrm{CH}_{1}\right)$ for sensing the PU signal, and the 1st SU in the 3rd cluster can utilize the rigid reporting time slots of the previous 1st cluster head $\left(\mathrm{CH}_{1}\right)$ and 2nd cluster head $\left(\mathrm{CH}_{2}\right)$ for sensing the PU signal, and so on. 


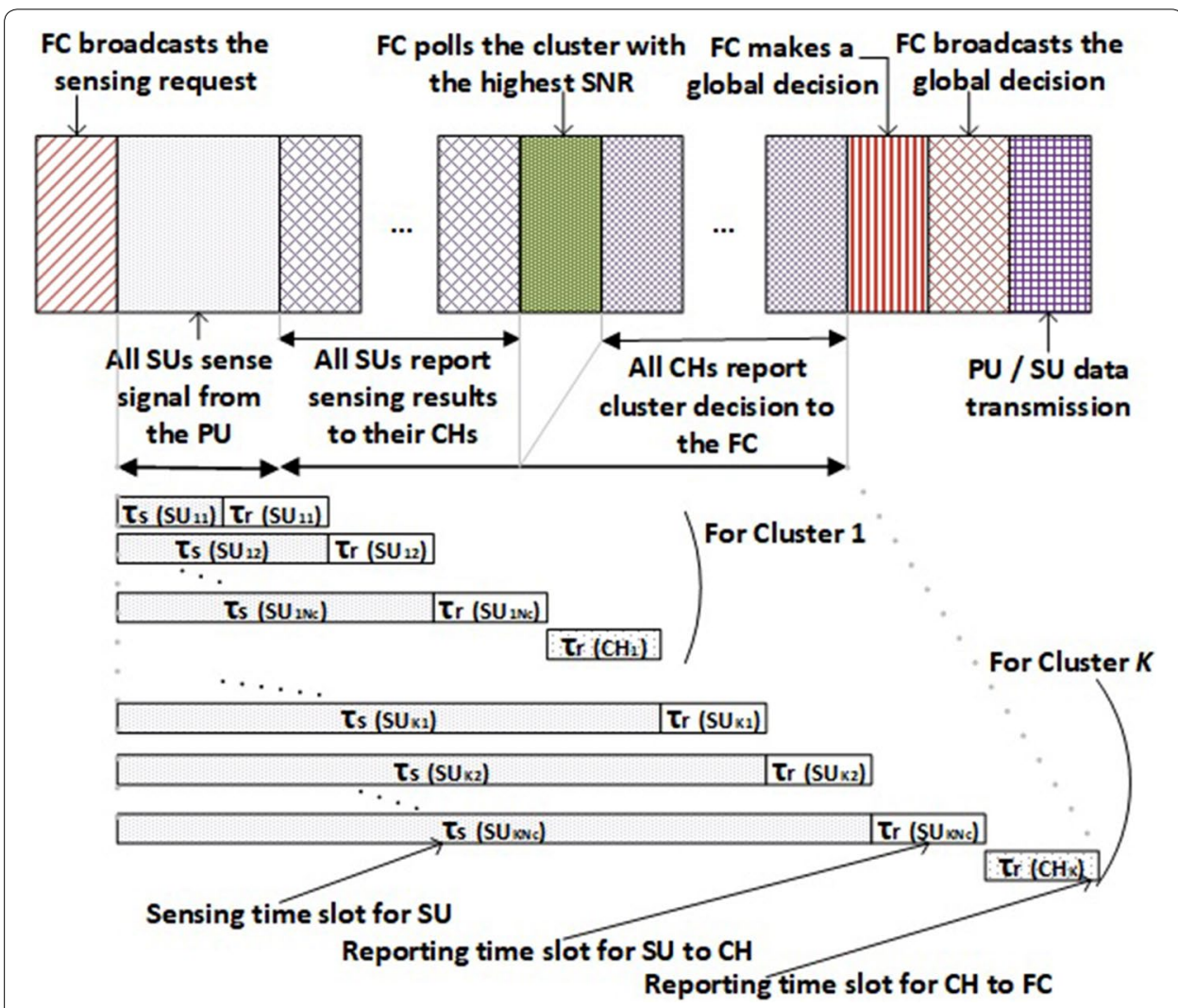

Fig. 4 Frame structure of a time slot for sensing, reporting and packet transmission in the proposed sequential approach in the CCRRN utilizing the reporting framework

Lemma 2 When the PU signal $x(l)$ is a BPSK-modulated signal, $w_{j}(l)$ is a CSCG channel noise between the PU and the jth SU and $z_{j}(l)$ is the reporting channel noise between the jth SU and the kth CH which is defined as CSCG. An estimation of the received signal power is given by all SUs in the proposed sequential approach with a relay as given by

$$
\begin{cases}\aleph\left(L \sigma_{w+z}^{2},\right. & \left.L \sigma_{w+z}^{4}\right) \\ \aleph\left(L\left(1+P_{t}|h|^{2}|g|^{2} \gamma\right) \sigma_{w+z}^{2},\right. & \left.L\left(1+2 P_{t}|h|^{2}|g|^{2} \gamma\right) \sigma_{w+z}^{4}\right)\end{cases}
$$

where $\sigma_{w+z}^{2}=\sum_{j=1}^{N}\left(\sigma_{w, j}^{2}+\sigma_{z, j}^{2}\right), \sigma_{w+z}^{4}=\sum_{j=1}^{N}\left(\sigma_{w, j}^{4}+\sigma_{z, j}^{4}\right)$, and $\gamma=\sum_{j=1}^{N} \gamma_{j}$

Proof Please see Appendix.

Proposition 2 In the proposed sequential approach in the CCRRN with and without a relay, all the SUs at the kth clusters have obtained the longer/flexible sensing time slot which is denoted as follows

$$
\tau_{s}^{k j}=\tau_{s}+(j-1) \tau_{r, S U}+(k-1) \tau_{r, C H}
$$


where $\tau_{s}^{k j}$ denotes the longer/flexible sensing time slot for the jth SU at the kth cluster, $\tau_{r, S U}$ denotes the reporting time slot for each SU and $\tau_{r, C H}$ denotes the reporting time slot for each $\mathrm{CH}$.

Proof Please see Appendix.

Based on the Eq. (15), we can calculate the probability of false alarm and the probability of detection in the proposed sequential approach with a relay at the $k$ th cluster as follows

$$
\begin{aligned}
P_{f, k}^{\text {prop }} & =\operatorname{Pr}\left[C_{k} \geq \lambda_{k}^{\text {prop }} \mid H_{0}\right] \\
& =Q\left(\left(\frac{\lambda_{k}^{\text {prop }}}{\sigma_{w+z}^{2}}-1\right) \sqrt{\tau_{s}^{k j} F_{s}}\right) \\
P_{d, k}^{\text {prop }} & =\operatorname{Pr}\left[C_{k} \geq \lambda_{k}^{\text {prop }} \mid H_{1}\right] \\
& =Q\left(\left(\frac{\lambda_{k}^{\text {prop }}}{\sigma_{w+z}^{2}}-P_{t}|h|^{2}|g|^{2} \gamma-1\right) \sqrt{\frac{\tau_{s}^{k j} F_{s}}{\left(1+2 P_{t}|h|^{2}|g|^{2} \gamma\right)}}\right)
\end{aligned}
$$

From Eqs. (16) and (17), we can calculate the decision threshold, $\lambda_{k}^{\text {prop }}$ at the $k$ th $\mathrm{CH}$ as follows

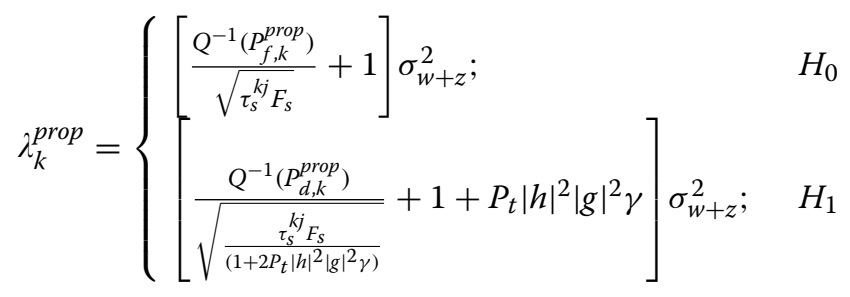

At the FC, all cluster decisions received will be combined to make a global decision $\left(P_{f, F C}^{m} / P_{d, F C}^{m}\right)$ about the presence or absence of the PU signal by using the $n-$ out - of $-k$ rule test as follows

$$
\begin{aligned}
& P_{f, F C}^{m}=\sum_{j=\beta}^{K} \sum_{A(j) \subset(1, \ldots, K)}\left[\prod_{k \subset A(j)} P_{f, k}^{m} \prod_{k \not \subset A(j)}\left(1-P_{f, k}^{m}\right)\right] \\
& P_{d, F C}^{m}=\sum_{j=\beta}^{K} \sum_{A(j) \subset(1, \ldots, K)}\left[\prod_{k \subset A(j)} P_{d, k}^{m} \prod_{k \not \subset A(j)}\left(1-P_{d, k}^{m}\right)\right]
\end{aligned}
$$

where the second summation with a subscript of $A(j) \subset 1,2, \ldots, K$ denotes the sum of all possible subsets with the $j$ th SUs in the $k$ th cluster and $\beta$ denotes the global threshold at the FC. Moreover, $m$ stands for index $(0,1,2)$ here, $m=0$ indicates the conventional non-sequential approach with no relay, $m=1$ indicates the proposed sequential approach with a relay and $m=2$ indicates the proposed sequential approach with no relay. 


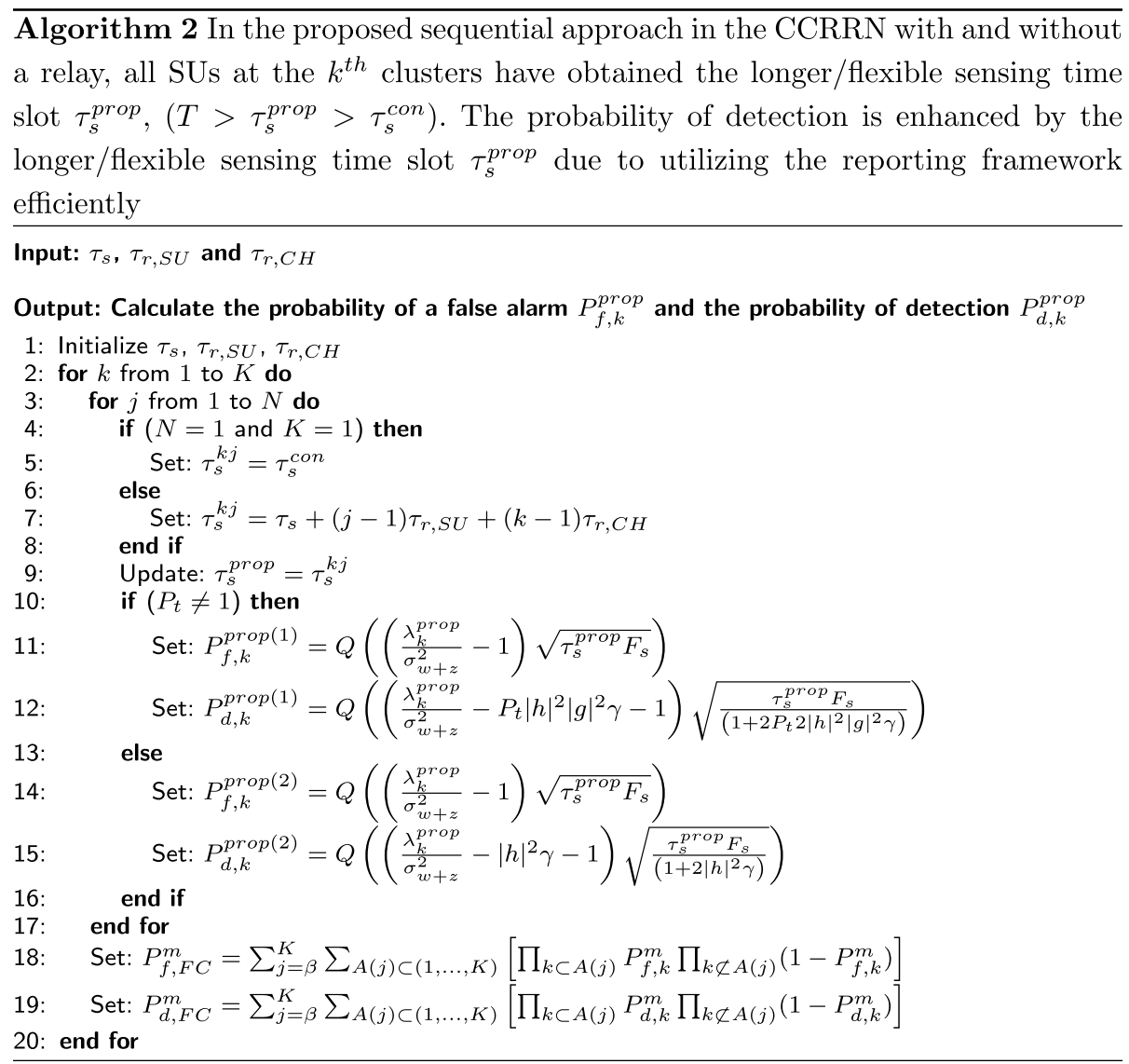

The proposed Algorithm 2 exhibits the whole idea. In Algorithm 2, it checks $N=1, K=1$ (see line 4) as the 1 st SU of the 1 st cluster in this case the reporting framework is not being utilized as it is obtaining a fixed/rigid sensing time slot (see line 5); otherwise, all SUs are utilizing the reporting framework and are obtaining a longer/flexible sensing time slot (see line 7). Then, it computes the probability of false alarm (see line 11) and the probability of detection (see line 12) at the $k$ th cluster in the proposed sequential approach with a relay $\left(P_{t} \neq 1\right)$ (see line 10), otherwise, it computes the probability of false alarm (see line 14) and the probability of detection (see line 15) at the $k$ th cluster in the proposed sequential approach with no relay $\left(P_{t}=1\right)$. After then, it computes a global decision at the FC which is defined as $\left(P_{f, F C}^{m} / P_{d, F C}^{m}\right)$ (see line 18/line 19).

The network end-to-end delay is another important factor in the CRN [30]. Based on Figs. 3 and 4, the network end-to-end delay of the conventional CCRRN with no relay and the proposed CCRRN with and without a relay are the same, denoted as $\delta_{d}$ which is defined as follows

$$
\begin{aligned}
\delta_{d} & =N \tau_{r, S U}+K \tau_{r, C H} \\
& =(N+K) \tau_{r}
\end{aligned}
$$

Here, if $\tau_{r, S U}=\tau_{r, C H}$, then the reporting time slot is denoted as $\tau_{r}$. 


\section{Sum rate analysis}

With the frame structure and sensing performance in the above section, we can analyze the system sum rate with several assumptions. In the transmission slot, if the SU transmitter does not detect the PU signal, it determines that the channel is available and transmits data to its own receiver; otherwise, it waits until the channel becomes available for its transmission that are scheduled in a round-robin manner.

When sensing accuracy perfect, e.g. when the PU is absent and the absence of the $\mathrm{PU}$ is accurately detected by the $\mathrm{SU}$, the $\mathrm{SU}$ can access the primary spectrum with the probability $\left(1-P_{f, F C}^{m}\right)$; otherwise, when the PU is present and the presence of the PU is accurately detected by the $\mathrm{SU}$, the $\mathrm{SU}$ can not access the primary spectrum with the probability $P_{d, F C}$. In this case, the sum rate of all users including both PU and SUs in a round-robin manner is calculated as follows $[36,37]$

$$
R_{\text {sum }}=\rho P_{d, F C}^{m} C_{P U}+(1-\rho)\left(1-P_{f, F C}^{m}\right) C_{j, S U}
$$

where $C_{P U}$ denotes the channel capacity of the PU link, $C_{j, S U}$ denotes the channel capacity of the $j$ th SU link, and $\rho \in[0,1]$ denotes the primary activity factor which means the probability of the PUs transmitting in a given frame.

The $C_{p}$ and $C_{j, S U}$ are given as follows

$$
\begin{aligned}
& C_{P U}=\log _{2}\left(1+S N R_{P U}\right) \\
& C_{j, S U}=\frac{T-\tau_{s}^{\text {prop }}}{T} \log _{2}\left(1+S N R_{j, S U}\right)
\end{aligned}
$$

where $S N R_{P U}$ denotes the SNR of the PUs link, $S N R_{j, S U}$ denotes the SNR of the jth SU link in the CCRRN and $T$ denotes the total frame length.

Lemma 3 If the optimal probability of false alarm $P_{f, k}^{*}$ is a non-decreasing function of $\rho$, then the $P_{f, k}^{*}$ is maximizing the sum rate as follows

$$
\max _{P_{f, k}=P_{f, k}^{*}} R_{\text {sum }}
$$

Proof Please see Appendix.

\section{Simulation results and discussion}

In this section, we verify the theoretical results and evaluate the performance of the proposed sequential approach with and without a relay. This is done through numerical simulations via Mathlab.

Monte-Carlo simulations were carried out using the simulation parameters listed in Table 2 below.

Figures 5, 6 and 7 respectively show cluster and global sensing performance, receiver operating characteristic (ROC) curves for the conventional non-sequential approach with no relay, the proposed sequential approach with no relay and the proposed sequential approach with a relay. In the conventional non-sequential approach with no relay, 
Table 2 Simulation parameters

\begin{tabular}{ll}
\hline Parameters & Value \\
\hline The total number of $S U s, N$ & 12 \\
The total number of $\mathrm{CHs}, K$ & 3 \\
The sampling frequency, $F_{S}$ & $300 \mathrm{kHz}$ \\
The sensing time, $\tau_{s}$ & $300 \mathrm{~ms}$ \\
The reporting time at the $\mathrm{SU}, \tau_{r, S U}$ & $30 \mathrm{~ms}$ \\
The reporting time at the $\mathrm{CH}, \tau_{r, C H}$ & $30 \mathrm{~ms}$ \\
The PUs signal, $x(l)$ & BPSK \\
The channel noise, $w(I)$ and the reporting channel noise, $Z(l)$ & $\mathrm{CSCG}$ \\
The $S N R$ of $\mathrm{PU}, S N R P U$ & $10 \mathrm{~dB}$ \\
\hline
\end{tabular}

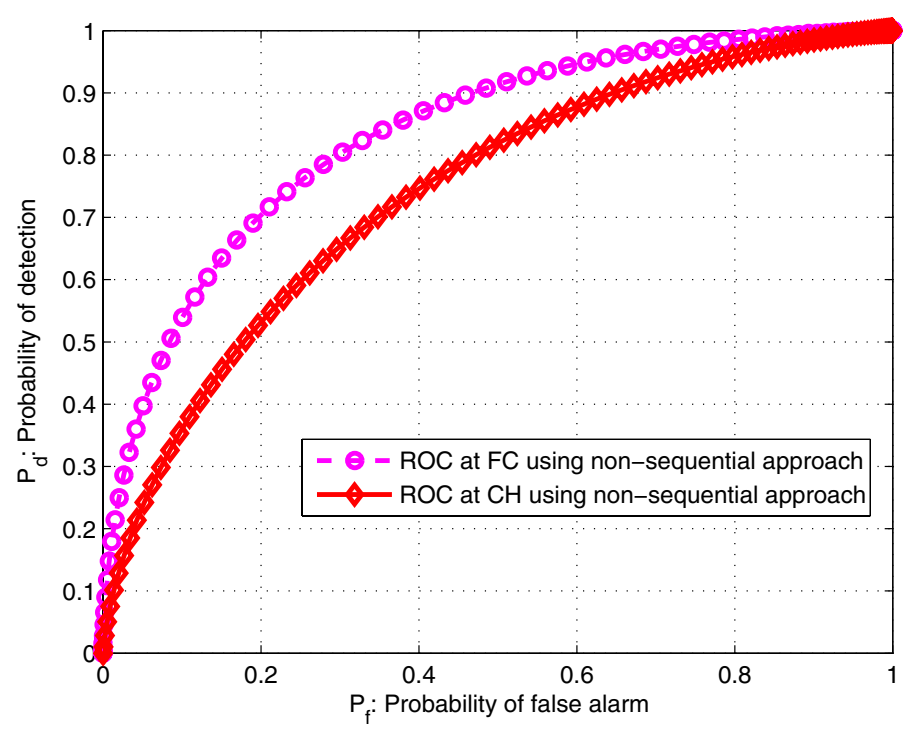

Fig. 5 ROC curves at $\mathrm{CH}$ and $\mathrm{FC}$ for the conventional non-sequential approach with no relay

$3 \mathrm{CHs}$ have the same performance, then just one curve is shown in Fig. 5. The reason is that the rigid sensing time $\tau_{s}^{k j}$ in the Eq. (13) for the case of the conventional nonsequential approach has no contribution on sensing gain, as mentioned above. As shown in Fig. 6, the third cluster has a better sensing performance than the others because the SUs in this cluster have a longer sensing duration than those in the other first and second clusters due to the proposed sequential approach in Eq. (15) for the case that the reporting time has contributed to sensing gain, as mentioned above. As shown in Fig. 7, the third cluster has a much better sensing performance than the others because the SUs in this cluster have a longer sensing duration due to the sequential approach than those in the other first and second clusters. The reasons are firstly, the flexible sensing time $\tau_{s}^{k j}$ in Eq. (15) has contributed to the sensing gain; and secondly, each SU acts in an AF manner in the proposed sequential approach with a relay based on Eqs. (16) and (17), as mentioned above.

As shown in Fig. 8, by comparing these ROCs at the FC based on Eqs. (19) and (20) for the conventional non-sequential approach with no relay, the proposed sequential 


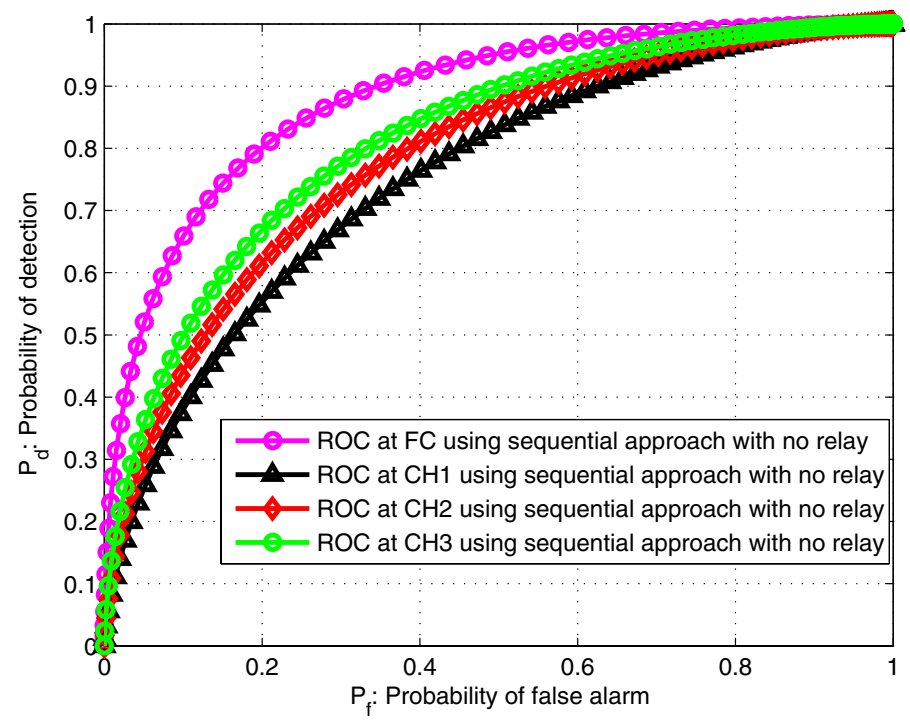

Fig. 6 ROC curves at $\mathrm{CH}$ and FC for the proposed sequential approach with no relay

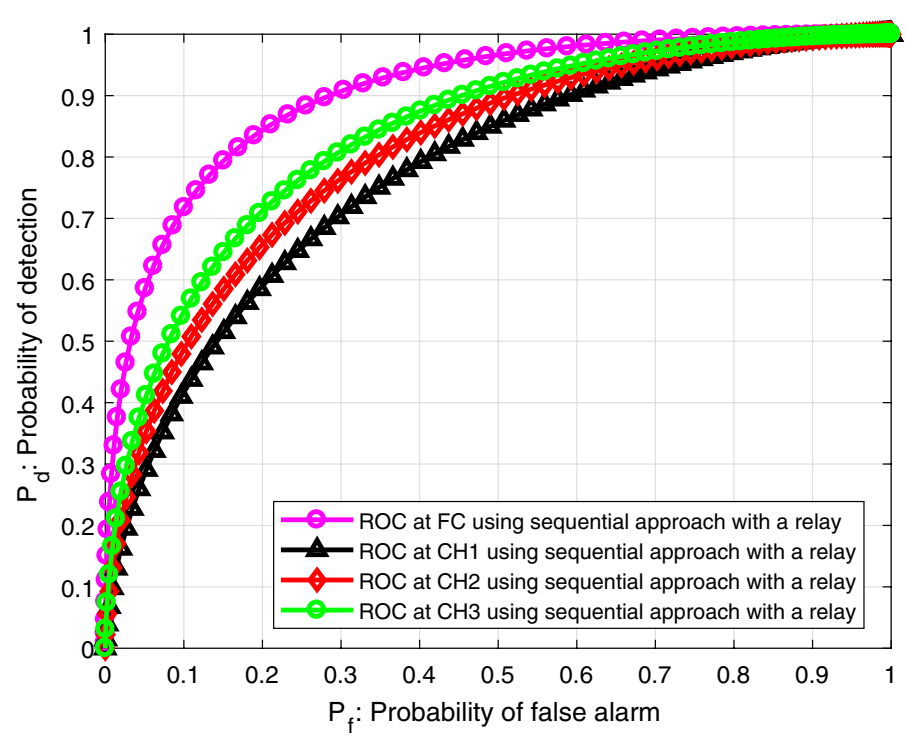

Fig. $7 \mathrm{ROC}$ curves at $\mathrm{CH}$ and $\mathrm{FC}$ for the proposed sequential approach with a relay

approach with no relay and proposed sequential approach with a relay, it can be seen that the proposed sequential approach with and without a relay shows better sensing capabilities than the conventional non-sequential approach with no relay which can be applicable for the future IoT systems. In addition, the proposed sequential approach with a relay shows much better sensing performance than the proposed sequential approach with no relay because each SU simply acts as a relay in the proposed sequential approach with a relay. It is found that the proposed sequential approach with a relay outperforms the other approaches for all cases which makes it more applicable for the future IoT systems. 


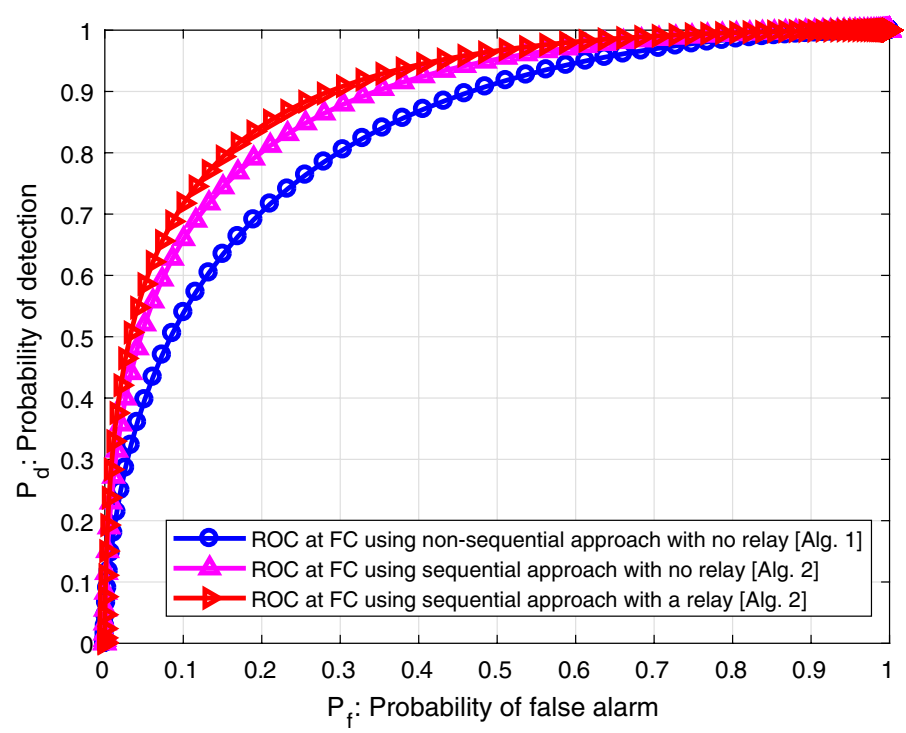

Fig. 8 ROC curves at FC for the conventional non-sequential approach with no relay, the proposed sequential approach with no relay and the proposed sequential approach with a relay

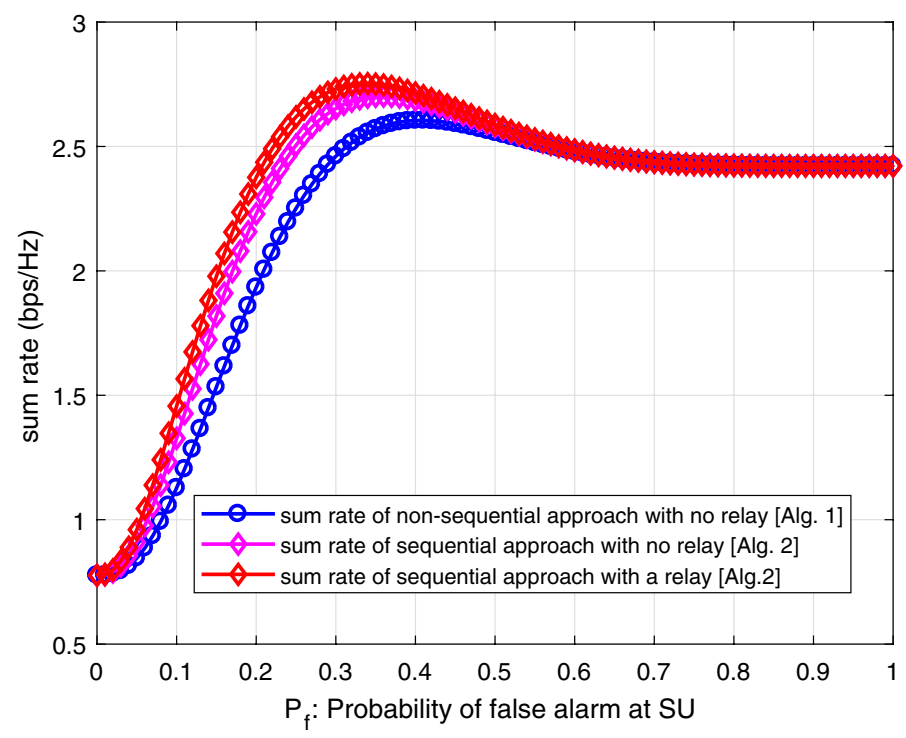

Fig. 9 Sum rate curves vs. probability of false alarm $P_{f}$ for the SU in the conventional non-sequential approach with no relay, the sequential approach with no relay and the sequential approach with a relay when the primary activity factor is $\rho=0.7$

Figure 9 shows the sum rates for the conventional non-sequential approach with no relay, the proposed sequential approach with no relay and the proposed sequential approach with a relay depending on the false alarm probability of a SU, i.e., the sum rate is a function of $P_{f}$. The sum rate of the proposed sequential approach with a relay is higher than when compared with both the conventional non-sequential approaches and the sequential approach with no relay for the entire range of $P_{f}$, which can be more applicable for the future IoT. 


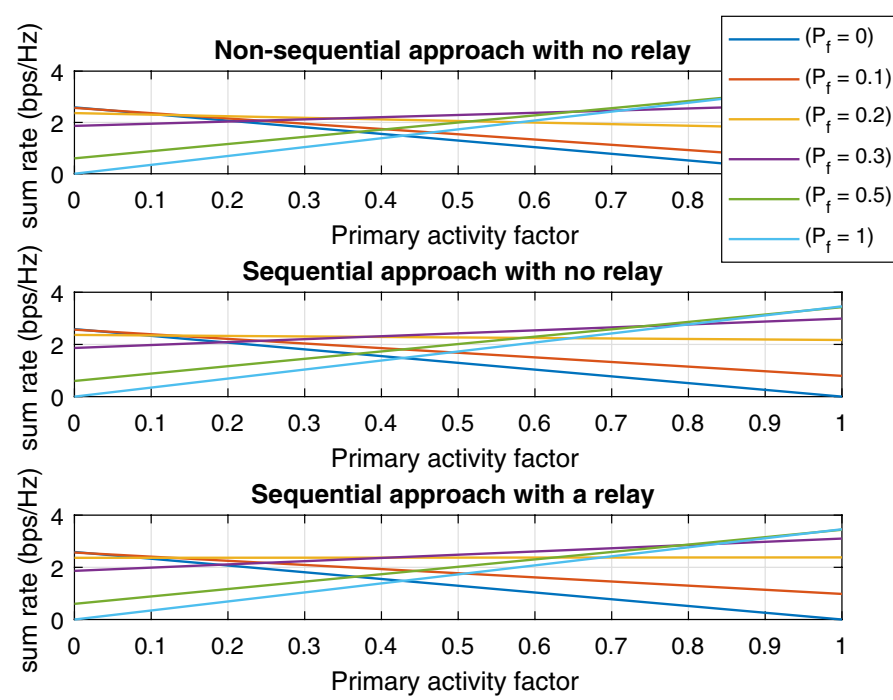

Fig. 10 Sum rate curves vs. the primary activity factor $\rho$ for the PU in the conventional non-sequential approach with no relay, the sequential approach with no relay and the sequential approach with a relay when the entire probability of false alarm $P_{f}$

Moreover, the sum rate curve is a function of $\rho$ for a given probability of false alarm $\left(P_{f}=0,0.1,0.2,0.3,0.5,1\right)$. Therefore, we show the sum rate of the conventional nonsequential approach with no relay, the proposed sequential approach with no relay and the proposed sequential approach with a relay, respectively as shown in Fig. 10.

Additionally, the sum rate curve is a quasi-concave function of $P_{f}$ for a given primary activity factor $\rho$. Therefore, there exists the optimal value of $P_{f}$ which enhances the sum rate for a given $\rho$. For the sum rate of the proposed sequential approach with a relay when $\rho=0.8$, the optimal $P_{f}$ is given by 0.38 . In the case of the proposed sequential approach with no relay and the conventional non-sequential approach with no relay respectively, the optimal probability of false alarm $P_{f}^{*}$ is 0.41 and 0.47 as shown in Fig. 11.

In order to compare the sensing gain at $\mathrm{CH}_{3}$, the proposed sequential approach with a relay and with no relay, can detect the spectrum with $72 \%$ and $68 \%$ detection accuracy, respectively whereas the conventional non-sequential approach with no relay detects the PU's signal with $54 \%$ as shown in Table 3.

In order to compare the sensing gain at a FC, the proposed sequential approach with a relay and with no relay can detect the spectrum with $84 \%$ and $80 \%$ detection accuracy, respectively whereas the conventional non-sequential approach with no relay detects the PU's signal with $70 \%$ as shown in Table 4.

In order to compare the sum rate, the proposed sequential approach with no relay and with a relay can be obtained as an enhanced sum rate with $2.62 \mathrm{~Hz}$ and $2.75 \mathrm{~Hz}$, respectively compared to the conventional non-sequential approach with no relay is $2.50 \mathrm{~Hz}$ as shown in Table 5.

In order to compare the optimal probability of false alarm $\left(P_{f}^{*}\right)$, the proposed sequential approach with no relay and with a relay achieves an accuracy with respect to false alarm of $41 \%$ and $39 \%$, respectively, compared to the conventional non-sequential approach with no relay which achieves an accuracy of $49 \%$ as shown in Table 6. 


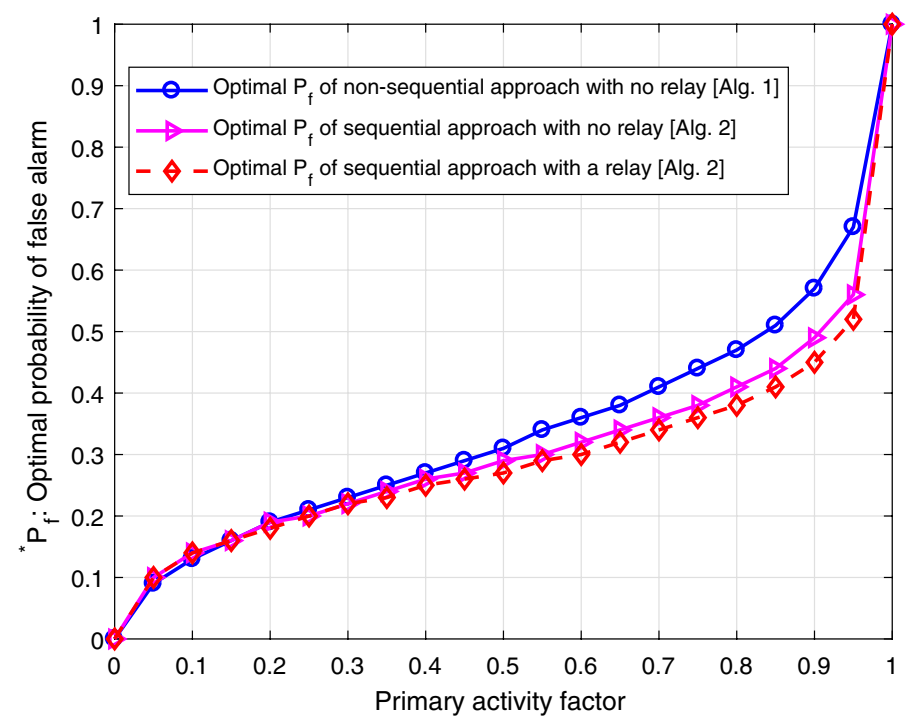

Fig. 11 Optimal probability of false alarm $P_{f}^{*}$ vs the primary activity factor $\rho$ in the conventional non-sequential approach with no relay, the sequential approach with no relay and the sequential approach with a relay

Table 3 Different approaches vs. the profferent approaches vs. the probability ofbability of detection and probability of false alarm

\begin{tabular}{llllllllllll}
\hline Approaches & \multicolumn{4}{c}{ Probability of detection $\boldsymbol{P}_{\boldsymbol{d}}$} & \multicolumn{6}{c}{ Probability of false alarm $\boldsymbol{P}_{\boldsymbol{f}}$} & Figures \\
\hline Conventional approach & $\mathrm{CH}_{1}$ & $\mathrm{CH}_{2}$ & $\mathrm{CH}_{3}$ & $\mathrm{FC}$ & $\mathrm{CH}_{1}$ & $\mathrm{CH}_{2}$ & $\mathrm{CH}_{3}$ & $\mathrm{FC}$ & Fig. 5 \\
& 0.54 & 0.54 & 0.54 & 0.70 & 0.20 & 0.20 & 0.20 & 0.20 & \\
Proposed approach with no relay & 0.58 & 0.62 & 0.68 & 0.80 & 0.20 & 0.20 & 0.20 & 0.20 & Fig. 6 \\
Proposed approach with a relay & 0.59 & 0.68 & 0.72 & 0.84 & 0.20 & 0.20 & 0.20 & 0.20 & Fig. 7 \\
\hline
\end{tabular}

Table 4 Sensing gain at the FC vs. different approaches

\begin{tabular}{lllll}
\hline Items & Conventional approach & Prop. approach with no relay & Prop. approach with a relay & Figure \\
\hline$P_{d}$ & 0.70 & 0.80 & 0.84 & Fig. 8 \\
$P_{f}$ & 0.20 & 0.20 & 0.20 & \\
\hline
\end{tabular}

Table 5 The sum rate vs. the probability of false alarm

\begin{tabular}{lllll}
\hline Items & Conventional approach & Prop. approach with no relay & Prop. approach with a relay & Figure \\
\hline Sum rate & 2.50 & 2.62 & 2.75 & Fig. 9 \\
$P_{f}$ & 0.31 & 0.31 & 0.31 & \\
\hline
\end{tabular}

Table 6 The optimal probability of false alarm vs. primary activity factor

\begin{tabular}{lllll}
\hline Items & Conventional approach & Prop. approach with no relay & Prop. approach with a relay & Figure \\
\hline$P_{f}^{*}$ & 0.49 & 0.41 & 0.39 & Fig. 11 \\
$\rho$ & 0.80 & 0.80 & 0.80 & \\
\hline
\end{tabular}


The results listed in Tables 3, 4, 5 and 6 show that the proposed sequential approach with a relay achieves better sensing gain, enhanced sum rates and lower optimal probability of false alarm $P_{f}^{*}$ compared to the conventional non-sequential approach with no relay which is more applicable for the future IoT.

\title{
Conclusion and future works
}

The main purpose of the proposed sequential approach in the CCRRN with and without a relay is to achieve not only better sensing gain of SUs, but also to maximize the sum rate of the SU's transmitter and receiver. In this paper, an efficient reporting mechanism scheme based on the sequential approach in the CCRRN has been proposed to gain a better detection and enhanced sum rate by utilizing the reporting frameworks of SUs and CHs. In detection gain, the probability of detection in the proposed sequential approach in the CCRRN with a relay are $4 \%$ and $14 \%$ over the proposed sequential approach in the CCRRN with no relay and the conventional non-sequential approach in the CCRRN with no relay, respectively. In addition, the proposed sequential approach in the CCRRN with no relay and with a relay achieved an optimal probability of false alarm $P_{f}^{*}$ which maximizes the sum rate for a given primary activity factor $\rho$ over the conventional non-sequential approach in the CCRRN with no relay. In sum rate maximization, the sum rate of the proposed sequential approach in the CCRRN with a relay are $13 \%$ and $25 \%$ over the proposed sequential approach in the CCRRN with no relay and the conventional non-sequential approach in the CCRRN with no relay, respectively. Therefore, we conclude that our proposed sequential approach in the CCRRN with a relay will be more applicable for the future IoT due to the fact that it mitigates the spectrum scarcity problem.

For future work, we will analyze the sum rate based on an efficient reporting mechanism whilst considering the interference to the PUs. Moreover, we will discuss the complexity of the proposed Algorithm 2 compared to the conventional Algorithm 1. Also, we will analyze detection and sum rate performance for scenarios when all SUs and $\mathrm{CHs}$ are moving.

\begin{abstract}
Abbreviations
CR: cognitive radio; SU: secondary user; CRN: cognitive radio network; PN: primary network; PU: primary user; CCRRN: cluster based cognitive radio relay network; loT: Internet of Things; NCSS: non-cooperative spectrum sensing; CSS: cooperative spectrum sensing; ED: energy detection; FC: fusion center; SDF: soft decision fusion; HDF: hard decision fusion; $\mathrm{CH}$ : cluster head; WSN: wireless sensor network; SBS: secondary base station; SU-RX: secondary receivers; AF: amplify and forward; TDMA: time division multiplexing access; LEACH-C: low energy adaptive clustering hierarchy-centralized; BPSK: binary phase shift keying; CSCG: circularly symmetric complex Gaussian; SNR: signal to noise ratio; ADC: analog to digital converter; PDF: probability distribution function; CLT: central limit theorem; LSC: linear statistic combination; ROC: receiver operating characteristic.
\end{abstract}

\section{Authors' contributions}

MS and EB provided the guideline to focus on issues, requiring solutions, and reviewed the overall manuscript. MSM conceived the study, drafting the article, revising it critically for intellectual content of the whole manuscript. They reviewed the technical contribution of the work and approved the final. All authors read and approved the final manuscript.

\section{Author details}

${ }^{1}$ Department of Information Technology, National University of Ireland Galway, University Road, Galway H91 TK33, Ireland. ${ }^{2}$ Department of Information and Communication Engineering, Islamic University, Shantidanga-Dulalpur, Kushtia 7003, Bangladesh.

\section{Authors information}

Md Sipon Miah received his BSc (Hon's), and MSc in Information and Communication Engineering (ICE) from the Islamic University (IU), Kushtia-7003, Bangladesh, in 2006 and 2007, respectively. Since 2010, he has been with the Department of Information and Communication Engineering (ICE), in the Islamic University (IU), Kushtia-7003, Bangladesh. He is 
currently an Associate Professor in the same Department. Sipon is currently pursuing a Structured Ph.D. in computer science in the Department of Information Technology (IT), National University of Ireland Galway (NUIG), Galway, Ireland. In 2016 Sipon was awarded the prestigious Hardiman Scholarship. His research interests include Spectrum Sensing, Energy Harvesting, MU-MIMO based Cognitive Radio Networks and Massive MIMO based Cognitive Radio Networks.

Dr. Michael Schukat is a lecturer and researcher in the Discipline of Information Technology at the National University of Ireland Galway (NUIG), Galway, Ireland. He is principal investigator of both the OSNA (Open Sensor Network Authentication) cyber security research group (http://www.osna-solutions.com) and the Performance Engineering Laboratory @ NUI Galway. His main research interests include security / privacy problems of connected real-time /time-aware embedded systems (i.e. industrial control, loT and cyber-physical systems) and their communication / time synchronisation protocols. He is actively involved in various security working groups on a European (e.g. COST Action Cryptacus) and International level (e.g. US-NIST CPS Public WG). Originally from Germany, Dr. Schukat studied Computer Science and Medical Informatics at the University of Hildesheim, where he graduated with a M.Sc. (Dipl. Inf.) in 1994 and a Ph.D. (Dr. rer. nat.) in 2000. Between 1994 and 2002 he worked in various industry positions where he specialised in deeply embedded real-time systems across diverse domains, such as industrial control, medical devices, automotive and network storage.

Dr. Enda Barrett is a Lecturer and researcher at the National University of Ireland Galway (NUIG), Galway, Ireland. In 2013, Enda received his Ph.D. in Computer Science from NUI Galway. His Ph.D. research investigated the application of a subset of machine learning techniques known as reinforcement learning to automate resource allocations and scale applications in infrastructure as a service cloud computing environments. Upon completion of his Ph.D., Enda joined Schneider Electric as a research engineer on a globally distributed innovation team. His main research interests include machine learning, distributed computing, cyber security and networking.

\section{Acknowledgements}

This work was supported in part by the Discipline of Information Technology (IT), National University of Ireland Galway (NUIG), Galway, Ireland and in part by the Hardiman Scholarship Postgraduate Research Foundation of National University of Ireland Galway (NUIG), Grant No. 16239003, Galway, Ireland.

\section{Competing interests}

The authors declare that they have no competing interests.

\section{Ethics approval and consent to participate}

This article does not contain any studies with human participants or animals performed by any of the authors.

\section{Publisher's Note}

Springer Nature remains neutral with regard to jurisdictional claims in published maps and institutional affiliations.

\section{Appendix}

Proof of Lemma 1 In the conventional non-sequential approach with no relay, the mean, $\mu_{1}$ of the received signal energy under the hypothesis $H_{1}$ is given as follows

$$
\begin{aligned}
\mu_{1} & =L\left(|h|^{2} p_{x}^{2}+\sigma_{w}^{2}\right) \\
& =L\left(\left(1+\frac{|h|^{2} p_{x}^{2}}{\sigma_{w}^{2}}\right) \sigma_{w}^{2}\right) \\
& =L\left(1+|h|^{2} \gamma\right) \sigma_{w}^{2}
\end{aligned}
$$

Similarly, the variance, $\sigma_{1}^{2}$ of the received signal energy under the hypothesis $H_{1}$ in the conventional non-sequential approach with no relay is given as follows [49]

$$
\sigma_{1}^{2}=L\left(E|x(l)|^{4}+E|w(l)|^{4}-\left(|h|^{2} p_{x}^{2}-\sigma_{w}^{2}\right)^{2}\right)
$$

If the PU signal is a complex M-ary pulse amplitude modulation (M-PAM) signal [50], then $E|x(l)|^{4}$ is given as follows

$$
E|x(l)|^{4}=\left(3-\frac{6}{5} \frac{M^{2}+1}{M^{2}-1}\right)|h|^{4} p_{x}^{4}
$$


We consider the BPSK modulated PU signal, in this case, we set $M=2$. By substituting this in Eq. (28) is given as follows

$$
E|x(l)|^{4}=|h|^{4} p_{x}^{4}
$$

Also, we consider the CSCG noise. We define $E|w(l)|^{4}$ as follows:

$$
E|w(l)|^{4}=2 \sigma_{w}^{4}
$$

We can rewrite the Eq. (27) using the Eqs. (29) and (30) as follows

$$
\begin{aligned}
\sigma_{1}^{2} & =L\left[|h|^{4} p_{x}^{4}+2 \sigma_{w}^{4}-\left(|h|^{4} p_{x}^{4}-2|h|^{2} p_{x}^{2} \sigma_{w}^{2}+\sigma_{w}^{4}\right)\right] \\
& =L\left(\sigma_{w}^{4}+2|h|^{2} p_{x}^{2} \sigma_{w}^{2}\right) \\
& =L\left(1+2|h|^{2} \frac{p_{x}^{2}}{\sigma_{w}^{2}}\right) \sigma_{w}^{4} \\
& =L\left(1+2|h|^{2} \gamma\right) \sigma_{w}^{4}
\end{aligned}
$$

Now, substituting the value of the PU signal, $p_{x}^{2}=0$, then we can calculate the mean, $\mu_{0}$ is given under the hypothesis $H_{0}$ in the conventional non-sequential approach with no relay as follows

$$
\mu_{0}=L \sigma_{w}^{2}
$$

Also, substituting the value of the PU signal, $p_{x}^{2}=0$ in Eq. (27), then we can calculate the variance, $\sigma_{0}^{2}$ is given under the hypothesis $H_{0}$ in the conventional approach with no relay as follows

$$
\begin{aligned}
\sigma_{0}^{2} & =L\left(E|w(l)|^{4}-\left(\sigma_{w}^{2}\right)^{2}\right) \\
& =L\left(2 \sigma_{w}^{4}-\sigma_{w}^{4}\right) \\
& =L \sigma_{w}^{4}
\end{aligned}
$$

Proof of Lemma 2 In the proposed sequential approach with a relay, we consider the reporting channel noise between the $j$ th SU and the $k$ th $\mathrm{CH}$ is $z(l)$. The mean, $\mu_{1}$ of the received signal energy under the hypothesis $H_{1}$ is given using Eq. (26) as follows

$$
\begin{aligned}
\mu_{1} & =L\left(P_{t}|h|^{2}|g|^{2} p_{x}^{2}+\sigma_{w+z}^{2}\right) \\
& =L\left(\left(1+\frac{P_{t}|h|^{2}|g|^{2} p_{x}^{2}}{\sigma_{w+z}^{2}}\right) \sigma_{w+z}^{2}\right) \\
& =L\left(1+P_{t}|h|^{2}|g|^{2} \gamma\right) \sigma_{w+z}^{2}
\end{aligned}
$$

Similarly, in the proposed sequential approach with a relay, the variance, $\sigma_{1}^{2}$ of the received signal energy under the hypothesis $H_{1}$ with relay is given using Eq. (27) as follows

$$
\sigma_{1}^{2}=L\left(E|x(l)|^{4}+E|w(l)+z(l)|^{4}-\left(P_{t}|h|^{2}|g|^{2} p_{x}^{2}-\sigma_{w+z}^{2}\right)^{2}\right)
$$

In the proposed sequential approach with a relay, if the PU signal is a complex $\mathrm{M}$-ary pulse amplitude modulation (M-PAM) signal, then $E|x(l)|^{4}$ is given using Eq. (28) as follows

$$
E|x(l)|^{4}=\left(3-\frac{6}{5} \frac{M^{2}+1}{M^{2}-1}\right) P_{t}^{2}|h|^{4}|g|^{4} p_{x}^{4}
$$


In the proposed sequential approach with a relay, we consider the BPSK modulated PU signal, in this case, we set $M=2$. By substituting this in Eq. (36) is given as follows

$$
E|x(l)|^{4}=P_{t}^{2}|h|^{4}|g|^{4} p_{x}^{4}
$$

Also, in the proposed sequential approach with a relay, we consider the CSCG noise. We define $E|w(l)+z(l)|$ as follows

$$
E|w(l)+z(l)|^{4}=2 \sigma_{w+z}^{4}
$$

We can rewrite the Eq. (35) using the Eqs. (37) and (38) as follows

$$
\begin{aligned}
\sigma_{1}^{2} & =L\left[P_{t}^{2}|h|^{4}|g|^{4} p_{x}^{4}+2 \sigma_{w+z}^{4}-\left(P_{t}^{2}|h|^{4}|g|^{4} p_{x}^{2} \sigma_{w+z}^{2}-2 P_{t}|h|^{2}|g|^{2} p_{x}^{2} \sigma_{w+z}^{2}+\sigma_{w+z}^{4}\right)\right] \\
& =L\left(\sigma_{w+z}^{4}+2 P_{t}|h|^{2}|g|^{2} p_{x}^{2} \sigma_{w+z}^{2}\right) \\
& =L\left(\sigma_{w+z}^{4}+2 P_{t}|h|^{2}|g|^{2} p_{x}^{2} \sigma_{w+z}^{2}\right) \\
& =L\left(1+2 P_{t}|h|^{2}|g|^{2} \frac{p_{x}^{2}}{\sigma_{w+z}^{2}}\right) \sigma_{w+z}^{4} \\
& =L\left(1+2 P_{t}|h|^{2}|g|^{2} \gamma\right) \sigma_{w+z}^{4}
\end{aligned}
$$

Now, substituting the value of the PU signal, $p_{x}^{2}$, then we can calculate the mean, $\mu_{0}$ is given under the hypothesis $H_{0}$ in the proposed sequential approach with a relay as follows

$$
\mu_{0}=L \sigma_{w+z}^{2}
$$

Also, substituting the value of the PU signal, $p_{x}^{2}=0$ in Eq. (35), then we can calculate the variance, $\sigma_{0}^{2}$ is given under the hypothesis $H_{0}$ in the proposed sequential approach with a relay as follows

$$
\begin{aligned}
\sigma_{0}^{2} & =L\left[E|w(l)+z(l)|^{4}-\left(\sigma_{w+z}^{2}\right)^{2}\right] \\
& =L\left(2 \sigma_{w+z}^{4}-\sigma_{w+z}^{4}\right) \\
& =L \sigma_{w+z}^{4}
\end{aligned}
$$

Proof of Lemma 3 First, we prove that the optimal probability of false alarm is a nondecreasing function of $\rho$ which is defined as $P_{f}^{*}(\rho)$. We can rewrite the Eqs. (16) and (17) as follows

$$
P_{d, k}^{\text {prop }}\left(P_{f, k}^{\text {prop }}\right)=Q\left[Q^{-1}\left(P_{f, k}^{\text {prop }}\right)-P_{t}|h|^{2}|g|^{2} \gamma \sqrt{\frac{\tau_{s}^{k j} F_{s}}{\left(1+2 P_{t}|h|^{2}|g|^{2} \gamma\right.}}\right]
$$

The first derivations of $R_{\text {sum }}$ in the Eq. (22) and for simplicity, we omits subscripts and superscripts as follows

$$
\begin{aligned}
& \frac{\partial R_{s u m}}{\partial P_{f}}=\rho \frac{\partial P_{d}\left(P_{f}\right)}{\partial P_{f}} C_{P U}+(1-\rho) \frac{\partial\left(1-P_{f}\right)}{\partial P_{f}} C_{S U} \\
& \frac{\partial R_{s u m}}{\partial P_{f}}=\rho \frac{\partial P_{d}\left(P_{f}\right)}{\partial P_{f}} C_{P U}-(1-\rho) C_{S U}=0 \\
& \left.\frac{\partial P_{d}\left(P_{f}\right)}{\partial P_{f}}\right|_{P_{f}=P_{f}^{*}}=\left(\frac{1}{\rho}-1\right) \frac{C_{S U}}{C_{P U}}
\end{aligned}
$$


Here, $\frac{\partial P_{d}\left(P_{f}^{*}\right)}{\partial P_{f}}$ is a monotone decreasing function of $\rho$. Therefore, the $P_{f}^{*}(\rho)$ is a nondecreasing function of $\rho$ due to the right side of Eq. (44) is also a monotonic decreasing function of $\rho$. The optimal probability of false alarm $P_{f}^{*}$ is maximizing the sum rate $R_{\text {sum }}^{\max }$.

Proof of Proposition 1 In the conventional non-sequential approach with no relay as shown in Fig. 3, it is observed that the $j$ th $\mathrm{SU}$ in the 1st cluster has a rigid sensing time slot to sense the PU signal as follows

$$
\tau_{s}^{1 j}=\tau_{s}
$$

Similarly, for the 2nd cluster, the sensing time for the jth SU can be obtained by

$$
\tau_{s}^{2 j}=\tau_{s}
$$

And so on. In summary, the $j$ th SU in the $k$ th cluster has the sensing time as follows

$$
\tau_{s}^{k j}=\tau_{s}=\tau_{s}^{c o n}
$$

Therefore, from the Eqs. (45), (46) and (47), it is obvious that the conventional nonsequential approach in the CCRRN underutilized the reporting framework.

Proof of Proposition 2 In the proposed sequential approach in the CCRRN as shown in Fig. 4 , it is observed that the 2 nd SU in the 1 st cluster have obtained flexible sensing time slot to sense the PU signal due to the 1st SU reporting time slot and the 2nd SU sensing time slot are merged to the 2nd SU sensing time slot as follows

$$
\tau_{s}^{12}=\tau_{s}^{12}+\tau_{r, S U}^{11}
$$

Similarly, for the 3rd SU in the 1st cluster have obtained as follows:

$$
\begin{aligned}
\tau_{s}^{13} & =\tau_{s}^{13}+\left[\tau_{r, S U}^{11}+\tau_{r, S U}^{12}\right] \\
& =\tau_{s}^{13}+\sum_{i=1}^{2} \tau_{r, S U}^{1 i}
\end{aligned}
$$

And so on. Finally, for the $j$ th SU in the 1st cluster have obtained as follows

$$
\begin{aligned}
\tau_{s}^{1 j} & =\tau_{s}^{1 j}+\left[\tau_{r, S U}^{11}+\tau_{r, S U}^{12}+\cdots+\tau_{r, S U}^{1(j-1)}\right] \\
& =\tau_{s}^{1 j}+\sum_{i=1} \tau_{r, S U}^{1 i} \\
& =\tau_{s}+(j-1) \tau_{r, S U}
\end{aligned}
$$

Similarly, for the 2nd cluster, the sensing time for the jth SU can be obtained by

$$
\begin{aligned}
\tau_{s}^{2 j} & =\tau_{s}^{2 j}+\left[\tau_{r, S U}^{21}+\tau_{r, S U}^{22}+\cdots+\tau_{r, S U}^{2(j-1)}\right]+\tau_{r, C H}^{1} \\
& =\tau_{s}^{2 j}+\sum_{i=1} \tau_{r, S U}^{1 i}+\tau_{r, C H}^{1} \\
& =\tau_{s}+(j-1) \tau_{r, S U}+\tau_{r, C H}^{1}
\end{aligned}
$$


From the Eqs. (48), (49), (50) and (51), we conclude that the $j$ th $S U$ in the $k$ th cluster has the sensing time as follows

$$
\begin{aligned}
\tau_{s}^{k j} & =\tau_{s}^{1 j}+\left[\tau_{r, S U}^{11}+\tau_{r, S U}^{12}+\cdots+\tau_{r, S U}^{1(j-1)}\right]+\left[\tau_{r, C H}^{1}+\tau_{r, C H}^{2}+\cdots+\tau_{r, C H}^{(k-1)}\right] \\
& =\tau_{s}^{1 j}+\sum_{i=1}^{j-1} \tau_{r, S U}^{1 i}+\sum_{q=1}^{k-1} \tau_{r, C H}^{q} \\
& =\tau_{s}+(j-1) \tau_{r, S U}+(k-1) \tau_{r, C H}
\end{aligned}
$$

Therefore, from the Eq. (52), it is obvious that the proposed sequential approach in the CCRRN utilizes the reporting framework, as it is obtaining a longer/flexible sensing time $\left(\tau_{s}^{k j}=\tau_{s}^{p r o p}\right)$ is equal to sum of the conventional fixed/rigid sensing time $\left(\tau_{s}^{c o n}\right)$, reporting time $\left(\tau_{r, S U}\right)$ at the $\mathrm{SU}$ and reporting time $\left(\tau_{r, C H}\right)$ at the $\mathrm{CH}$.

Received: 18 January 2018 Accepted: 22 May 2018

Published online: 07 June 2018

\section{References}

1. Mitola J, Maguire GQ (1999) Cognitive radio: making software radios more personal. IEEE Personal Commun 6(4):13-18. https://doi.org/10.1109/98.788210

2. Senthilmurugan S, Venkatesh TG (2017) Optimal channel sensing strategy for cognitive radio networks with heavytailed idle times. IEEE Trans Cogni Commun Netw 3(1):26-36. https://doi.org/10.1109/TCCN.2017.2669985

3. Afzal A, Zaidi SAR, Shakir MZ, Imran MA, Ghogho M, Vasilakos AV, McLernon DC, Qaraqe K (2015) The cognitive Internet of Things: a unified perspective. Mob Netw Appl 20(1):72-85. https://doi.org/10.1007/s1 1036-015-0583-6

4. Li T, Yuan J, Torlak M (2018) Network throughput optimization for random access narrowband cognitive radio Internet of things (NB-CR-IoT). IEEE Internet Things J 99:1-13. https://doi.org/10.1109/JIOT.2017.2789217

5. Park JH (2017) Advances in algorithm, multimedia, and network for future IT. J Inf Proces Syst 13(2):197-203. https:// doi.org/10.3745/JIPS.00.0004

6. Zhu J, Song Y, Jiang D, Song H (2017) A new deep-Q-learning-based transmission scheduling mechanism for the cognitive Internet of Things. IEEE Internet Things J 99:1-11. https://doi.org/10.1109/JIOT.2017.2759728

7. Yawada PS, Wei AJ (2016) Comparative study of spectrum sensing techniques base on techniques non-cooperative in cognitive radio networks. In: Proceedings of the 5 th international conference on computer science and network technology (ICCSNT). IEEE, Changchun, pp 517-520

8. Suseela B, Sivakumar D (2015) Non-cooperative spectrum sensing techniques in cognitive radio-a survey. In: 2015 IEEE technological innovation in ICT for agriculture and rural development (TIAR). IEEE, Chennai, pp 127-133

9. Nguyen VD, Shin OS (2018) Cooperative prediction-and-sensing-based spectrum sharing in cognitive radio networks. IEEE Trans Cogni Commun Netw 4(1):108-120

10. Rahman MA, Lee YD, Koo I (2016) An efficient transmission mode selection based on reinforcement learning for cooperative cognitive radio networks. Hum centric Comput Inf Sci 6(2):1-14. https://doi.org/10.1186/s1367 3-016-0057-2

11. Lv Q, Gao F (2015) Mathched filter based spectrum sensing and power level recognition with multiple antennas. In: 2015 IEEE China summit and international conference on signal and information processing (ChinaSIP). IEEE, Chengdu, pp 305-309

12. Enserink S, Cochran D (1994) A cyclostationary feature detector. In: Proceedings of the 28th asilomar conference on signals, systems and computers, vol. 2, pp 806-810

13. Anaand PP, Charan C (2016) Two stage spectrum sensing for cognitive radio networks using ED and AIC under noise uncertainty. In: 2016 IEEE international conference on recent trends in information technology. IEEE, Chennai, pp $1-6$

14. Miah MS, Rahman MM (2014) An eigenvalue and superposition approach based cooperative spectrum sensing in cognitive radio networks. In: 2014 IEEE international conference on electrical engineering and information and communication technology (ICEEICT). IEEE, Dhaka, pp 1-7

15. Mu J, Jing $X$, Huang H, Gao N (2018) Subspace-based method for spectrum sensing with multiple users over fading channel. IEEE Commun Lett 22(4):848-851

16. Chandrasekaran G, Kalyani K (2015) Performance analysis of cooperative spectrum sensing over $k-\mu$ shadowed fading. IEEE Wirel Commun Lett 4(5):553-556

17. Sun M, Zhao C, Yan S, Li B (2017) A novel spectrum sensing for cognitive radio networks with noise uncertainty. IEEE Trans Veh Technol 66(5):4424-4429

18. Chen Q, Motani M, Wong WC (2010) Cooperative spectrum sensing strategies with multiple relays. In: 2010 IEEE international conference on communication systems (ICCS). IEEE, Singapore, pp 540-544

19. Gupta M, Verma G, Dubey RK (2016) Cooperative spectrum sensing for cognitive radio based on adaptive threshold. In: Proceedings of the 2 nd international conference on computational intelligence and communication technology (CICT). IEEE, Ghaziabad, pp 444-448 
20. Homayounzadeh A, Mahdavi M (2015) Performance analysis of cooperative cognitive radio networks with imperfect sensing. In: 2015 IEEE international conference on communications, signal processing, and their applications (ICCSPA). IEEE, Sharjah, pp 1-6

21. Liao Y, Wang T, Song L, Jiao B (2014) Cooperative spectrum sensing for full-duplex cognitive radio networks. In: 2014 IEEE international communication systems (ICCS). IEEE, Macau, pp 56-60

22. Aysal TC, Kandeepan S, Piesiewicz R (2009) Cooperative spectrum sensing with noisy hard decision transmissions. In: IEEE international conference on communications (ICC'09). IEEE, Dresden, pp 1-5

23. Sun C, Zhang W, Letaief KB (2007) Cluster-based cooperative spectrum sensing in cognitive radio systems. In: IEEE international conference on communications (ICC'O7). IEEE, Glasgow, pp 2511-2515

24. Althunibat S, Denise BJ, Granelli F (2014) Secure cluster-based cooperative spectrum sensing against malicious attackers. In: Proceedings of IEEE globecom workshops. IEEE, Austin, pp 1284-1289

25. Rasheed T, Rashdi A, Akhtar AN (2015) A cluster based cooperative technique for spectrum sensing using relay factor. In: Proceedings of the 12th international Bhurban conference on applied sciences and technology (IBCAST). IEEE, Islamabad, pp 588-590

26. Singh R, Singh P, Duhan M (2014) An effective implementation of security based algorithmic approach in mobile adhoc networks. Hum centric Comput Inf Sci 4(1):7. https://doi.org/10.1186/s13673-014-0007-9

27. Pughat A, Sharma V (2015) A review on stochastic approach for dynamic power management in wireless sensor networks. Hum centric Comput Inf Sci 5(1):4. https://doi.org/10.1186/s13673-015-0021-6

28. Chen R, Park JM, Bian K (2008) Robust distributed spectrum sensing in cognitive radio networks. INFOCOM. IEEE, Phoenix, pp 1876-1884

29. Zou Q, Zheng S, Sayed AH (2010) Cooperative sensing via sequential detection. IEEE Trans Signal Proces 58(12):6266-6283

30. Xia W, Wang S, Liu W, Chen W (2009) Cluster-based energy efficient cooperative spectrum sensing in cognitive radios. In: Proceedings of the 5 th international conference on wireless communications, networking and mobile computing (WiCom'09). IEEE, Beijing, pp 1-4

31. Lee IH, Lee $H$ (2017) Achievable rate analysis for opportunistic non-orthogonal multiple access-based cooperative relaying systems. J Inf Proces Syst 13(3):630-642

32. Vu-Van H, Koo I (2014) A cluster-based sequential cooperative spectrum sensing scheme utilizing reporting framework for cognitive radios. IEEJ Trans Electr Electron Eng 9(3):282-287

33. Zhang W, Yang Y, Yeo CK (2015) Cluster-based cooperative spectrum sensing assignment strategy for heterogeneous cognitive radio network. IEEE Trans Veh Technol 64(6):2637-2647

34. Mashreghi M, Abolhassani B (2017) A cluster-based cooperative spectrum sensing strategy to maximize achievable throughput. Wirel Personal Commun 96(3):4557-4584

35. Tran H, Zepernick HJ, Phan H, Quang TP (2013) Cognitive cooperative networks with cluster-based relaying under interference constraints. In: Proceedings of the 5 th international conference on ubiquitous and future networks (ICUFN). IEEE, Da Nang, pp 542-546

36. Yu H (2013) Optimal channel sensing maximising sum rate in cognitive radio with multiple secondary links. Trans Emerg Telecommun Technol 24(7-8):777-784. https://doi.org/10.1002/ett.2725

37. Yu H (2013) Optimal channel sensing for heterogeneous cognitive networks: an analytical approach. KSII Trans Internet Inf syst 7(12):2987-3002

38. Miah MS, Schukat M, Barrett E (2017) Maximization of sum rate in AF-cognitive radio networks using superposition approach and n-out-of-k rule. In: Proceedings of the 28th Irish signals and systems conference (ISSC). IEEE, Killarney, pp 1-6

39. Heinzelman WB, Chandrakasan AP, Balakrishnan H (2002) An application-specific protocol architecture for wireless microsensor networks. IEEE Trans Wirel Commun 1(4):660-670. https://doi.org/10.1109/TWC.2002.804190

40. Sinha A, Lobiyal DK (2013) Performance evaluation of data aggregation for cluster-based wireless sensor network. Hum Centric Comput Inf Sci 3(1):13. https://doi.org/10.1186/2192-1962-3-13

41. Rahman MM, Miah MS, Sharmin D (2016) Cognitive improved LEACH (CoglLEACH) protocol for wireless sensor network. Trans Netw Commun 4(6):1. https://doi.org/10.14738/tnc.46.2301

42. Singh B, Lobiyal DK (2012) A novel energy-aware cluster head selection based on particle swarm optimization for wireless sensor networks. Hum Centric Comput Inf Sci 2(1):13. https://doi.org/10.1186/2192-1962-2-13

43. Urkowitz H (1967) Energy detection of unknown deterministic signals. Proc IEEE 55(4):523-531

44. Kostylev VI (2000) Characteristics of energy detection of quasideterministic radio signals. Radiophys Quantum Electron 43(10):833-839. https://doi.org/10.1023/A:1010387403443

45. Kostylev VI (2002) Energy detection of a signal with random amplitude. In: Proceedings of the IEEE international conference on communications (ICC'02). IEEE, New York, pp 1606-1610

46. Zhu J, Song Y, Jiang D, Song H (2016) Multi-armed bandit channel access scheme with cognitive radio technology in wireless sensor networks for the Internet of Things. IEEE Access 4:4609-4617

47. Ambede A, Vinod AP, Shreejith S (2017) Efficient FPGA implementation of a variable digital filter based spectrum sensing scheme for cognitive loT systems. In: Proceedings of the IEEE global Internet of things summit (GloTS). IEEE, Geneva, pp 1-4

48. Liu X, Xie JL (2017) Priority-based spectrum access in cognitive D2D networks for loT. In: Proceedings of the IEEE international conference on communications (ICC). IEEE, Paris, pp 1-6

49. Boyed S, Vandenberghe L (2004) Convex optimization. Cambridge University Press, Cambridge

50. Mathis $\mathrm{H}$ (2001) On the kurtosis of digitally modulated signals with timing offsets. In: Proceedings of the 3rd workshop on signal processing advances in wireless communications (SPAWC'01). IEEE, Taiwan, pp 86-89 